\title{
FEEDBACK IN STATE CONSTRAINED OPTIMAL CONTROL
}

\author{
Francis H. Clarke ${ }^{\dagger, 1}$, Ludovic Rifford ${ }^{1}$ And R.J. Stern*,2
}

\begin{abstract}
An optimal control problem is studied, in which the state is required to remain in a compact set $S$. A control feedback law is constructed which, for given $\varepsilon>0$, produces $\varepsilon$-optimal trajectories that satisfy the state constraint universally with respect to all initial conditions in $S$. The construction relies upon a constraint removal technique which utilizes geometric properties of inner approximations of $S$ and a related trajectory tracking result. The control feedback is shown to possess a robustness property with respect to state measurement error.
\end{abstract}

Mathematics Subject Classification. 49J24, 49J52, 49N55, 90D25.

Received December 19, 2000. Revised July 2, 2001.

\section{Contents}

1. Introduction 97

2. Preliminaries 99

2.1. Nonsmooth analysis 99

2.2. Extended dynamics 102

2.3. Discretized solutions 102

3. Inner approximations and state constrained trajectory tracking 103

3.1. Inner approximations 105

3.2. Signed distance function 109

3.3. State constrained trajectory tracking 110

3.4. Applications of the tracking lemma 113

3.5. Constraint removal 117

4. Near-optimal universal control feedback in state constrained optimal control 122

4.1. Main result 122

4.2. Robustness 128

References 132

Keywords and phrases: Optimal control, state constraint, near-optimal control feedback, nonsmooth analysis.

1 Institut Desargues, bâtiment 101, Université Claude Bernard Lyon I, 69622 Villeurbanne, France;

e-mail: clarke@desargues.univ-lyon1.fr

2 Department of Mathematics and Statistics, Concordia University, Montréal, Quebec H4B 1R6, Canada.

$\dagger$ Membre de l'Institut Universitaire de France.

* Research supported by the Natural Sciences Engineering Research Council of Canada and Le Fonds pour la Formation de Chercheurs et l'Aide à la Recherche du Québec. 


\section{INTRODUCTION}

Consider a control system

$$
\dot{x}(t)=f(x(t), u(t)) \quad \text { a.e. }
$$

where the state $x(\cdot)$ evolves in $\mathbb{R}^{n}$ and (open loop) controls $u(\cdot)$ are Lebesgue measurable functions $u: \mathbb{R} \rightarrow U$, where $U \subset \mathbb{R}^{m}$ is a compact control constraint set.

Suppose that $S \subset \mathbb{R}^{n}$ is a compact set which is weakly invariant (or in alternate terminology, viable or holdable); that is, for any $(\tau, \alpha) \in \mathbb{R} \times S$ there exists a control $u(\cdot)$ producing a (unique) trajectory $x(t):=x(t ; \tau, \alpha, u(\cdot))$ of $(1)$ with $x(\tau)=\alpha$ and $x(t) \in S$ for all $t \geq \tau$. Let $T>0$ be specified. For an initial phase $(\tau, \alpha) \in(-\infty, T] \times S$, consider the following Mayer problem $P(\tau, \alpha)$ with state constraint $S$ :

minimize $\ell(x(T))$

subject to

$$
\dot{x}=f(x, u) \text { a.e., } \quad x(\tau)=\alpha, \quad x(t) \in S \forall t \in[\tau, T]
$$

By a control feedback we simply mean any selection $k(t, x)$ of $U$; that is, $k: \mathbb{R} \times \mathbb{R}^{n} \rightarrow U$. Our central goal in the present work is the construction, for specified $\varepsilon>0$, of a control feedback which is $\varepsilon$-optimal in problem $P(\tau, \alpha)$, universally for initial phases in $[0, T] \times S$.

Even in very simple problems without state constraints, one cannot expect the existence of continuous control feedback laws which are optimal (or even near-optimal) universally; that is, for all initial phases in the set $[0, T] \times S$. Observe that continuity of $k$ is a minimal condition for the classical existence theory of ordinary differential equations to apply to $\dot{x}=f(x, k(t, x))$, so it is essential to work with a generalized solution concept; we use the system sampling concept, which is based on a discretization procedure. (The "performance gap" between continuous and discontinuous control feedbacks was studied in a differential games setting by Barabanova and Subbotin [2,3], Krasovskiu and Subbotin [27,28], and in a control context by Clarke et al. [15]; for further discussion see also Clarke et al. [12].)

Throughout this article, we shall assume that

- The cost functional $\ell: \mathbb{R}^{n} \rightarrow \mathbb{R}$ is locally Lipschitz,

and that the dynamics $f: S \times U \rightarrow \mathbb{R}^{n}$ satisfy the following standing hypotheses (where $\|\cdot\|$ denotes the Euclidean norm):

(F1) The function $f$ is continuous on $S \times U$ and is Lipschitz in the state variable $x$, uniformly for $u \in U$; that is, there exists $K$ such that

$$
\|f(x, u)-f(y, u)\| \leq K\|x-y\|
$$

whenever $(x, u)$ and $(y, u)$ are in $S \times U$.

(F2) The velocity set $f(x, U)$ is convex for every $x \in S$.

By standard "sequential compactness of trajectories" arguments (where condition (F2) as well as the weak invariance assumption come into play), the following facts are readily verified (see for example Clarke et al. [17]):

- The minimum in $P(\tau, \alpha)$ is attained; we denote this minimum by $V(\tau, \alpha)$, and refer to $V:(-\infty, T] \rightarrow \mathbb{R}$ as the value function.

- The value function is lower semicontinuous on $(-\infty, T] \times S$.

The value $V$ will be locally Lipschitz in the absence of a state constraint (i.e. when $S=\mathbb{R}^{n}$, in which case a growth hypothesis on the dynamics needs to be imposed). Essentially this is due to the local Lipschitzness of the attainability map as a function of the initial phase $(\tau, \alpha)$, which in turn follows from a "tracking" 
property for trajectories, akin to continuity of solutions with respect to initial data in the classical theory of ordinary differential equations; see e.g. [17]. On the other hand, when a state constraint is present, simple cases show that only lower semicontinuity of $V$ holds in general. Consider for example the problem with $f\left(x_{1}, x_{2}, u\right)=(0, u), U=[0,1]$,

$$
S=\left\{\left(x_{1}, 0\right): 0 \leq x_{1} \leq 1\right\} \cup\left\{\left(0, x_{2}\right):-1 \leq x_{2} \leq 0\right\},
$$

and where the cost is $\ell(x(1))=x_{2}(1)$. Then $S$ is obviously weakly invariant (since one can always apply the control $u \equiv 0)$. If $\left(x_{1}(0), x_{2}(0)\right)=(0,0)$, the minimum value in the problem is -1 , but for any other start point on the $x_{1}$-axis, the minimum is 0 . The reason for this lack of continuity of the state constrained value is that a property we can reasonably call "state constrained trajectory tracking" fails here. In particular, the trajectory $x(t)=(0,-t)$ emanating from $(0,0)$ cannot be approximated by a trajectory staying in $S$ and emanating from $\left(x_{1}(0), 0\right)$ with $x_{1}(0)>0$, no matter how small $x_{1}(0)$ is taken to be. This example shows that if continuity (or more) is required of $V$, as it will be in our subsequent results, the hypotheses on $S$ need to be strengthened beyond mere compactness and weak invariance, so that a suitable state constrained tracking property holds.

The layout of this article is as follows. Preliminaries on nonsmooth analysis, extended dynamics and discretized solutions are provided in Section 2. Then in Section 3, a state constrained tracking lemma is proven, when $S$ is wedged and the dynamics satisfy a strict inwardness condition with respect to the Clarke tangent cone to $S$. The tracking lemma extends one due to Soner [37]; it is distinguished from other generalizations of Soner's result which have appeared in the literature, because it is provided in a required uniform manner with respect to (sufficiently small) $r>0$ for each "inner approximation" $S_{r}$, consisting of those points whose distance to the complement of $S$ is not less than $r$. This family of inner approximations is shown to inherit relevant geometric properties posited for $S$. Consequences of the tracking lemma are obtained, involving continuity and convergence properties of the family of $S_{r}$-constrained value functions $V_{r}$, as well as results on constraint removal via a certain modification of the dynamics near the boundary of $S$; specifically we do so on the set $S \backslash S_{r}$. Due to the nice properties which we are able to establish for $V_{r}$, it transpires that the value function $\tilde{V}_{r}$ of the control problem with modified dynamics (and no state constraint) approximates $V$ (the value function in the $S$-constrained problem) uniformly on $[0, T] \times S$. While Section 3 is rather lengthy, the derived geometric properties of inner approximations will be seen to facilitate our constructive technique in the next section, and they will also prove useful in later work. In Section 4, we produce a near-optimal control feedback $k(t, x)$ whose discretized trajectories satisfy the state constraint, universally with respect to all initial phases in $[0, T] \times S$; our technique involves a judicious use of the quadratic inf-convolution of $\tilde{V}_{r}$, along with a resulting semiconcavity property. Concluding remarks deal with the robustness of $k(t, x)$ with respect to state measurement errors.

\section{Preliminaries}

Our general reference for this section is [17]; see also [13], Clarke [8, 9], Loewen [29] and Vinter [44].

\subsection{Nonsmooth analysis}

\subsubsection{Notation and definitions}

Here we will only present basic definitions and notation from nonsmooth analysis. Further required facts from nonsmooth analysis will be invoked below in an ad hoc manner. Most can be found in [17]; other references will be provided as required.

The Euclidean norm is denoted $\|\cdot\|$, and $\langle$,$\rangle is the usual inner product. The open unit ball in \mathbb{R}^{n}$ is denoted $B_{n}$, and we denote $\Sigma_{n}:=\left\{x \in \mathbb{R}^{n}:\|x\|=1\right\}$. The open ball of radius $\varepsilon$ centered at $x \in \mathbb{R}^{n}$ (that is, the set $\left.\left\{x+\varepsilon B_{n}\right\}\right)$ is denoted $B_{n}(x ; \varepsilon)$. For a set $Z \subset \mathbb{R}^{n}$, we denote by $\operatorname{co}(Z), \bar{Z}$, bdry $(Z)$, meas $(Z)$, and $\operatorname{int}(Z)$ the convex hull, closure, boundary, $n$-dimensional Lebesgue measure, and interior of $Z$, respectively. Also, we denote the closure of the complement of $Z$ by $\hat{Z}:=\operatorname{cl}\left\{\mathbb{R}^{n} \backslash Z\right\}$. The closed line segment between two points $x, y \in \mathbb{R}^{n}$ will be denoted $[x, y]$. 
Let $Z$ be a nonempty closed subset of $\mathbb{R}^{n}$. The distance of a point $u$ to $Z$ is denoted

$$
d_{Z}(u):=\min \{\|u-x\|: x \in Z\}
$$

The metric projection of $u$ on $Z$ is denoted

$$
\operatorname{proj}_{Z}(u):=\left\{x \in Z:\|u-x\|=d_{Z}(u)\right\}
$$

If $u \notin Z$ and $x \in \operatorname{proj}_{Z}(u)$, then the vector $u-x$ is called a perpendicular to $Z$ at $x$. The cone consisting of all nonnegative multiples of these perpendiculars is denoted $N_{Z}^{P}(x)$, and is referred to as the proximal normal cone (or P-normal cone) to $Z$ at $x$. If $x \in \operatorname{int}(Z)$ or no perpendiculars to $Z$ exist at $x$, then we set $N_{Z}^{P}(x)=\{0\}$. One can show that $N_{Z}^{P}(x) \neq\{0\}$ for a dense subset of $\operatorname{bdry}(Z)$.

Let $g: \mathbb{R}^{n} \rightarrow(-\infty, \infty]$ be an extended real valued function which is lower semicontinuous; that is, for each $x \in \mathbb{R}^{n}$,

$$
g(x) \leq \liminf _{y \rightarrow x} g(y)
$$

a property equivalent to closedness of the epigraph of $g$,

$$
\operatorname{epi}(g):=\{(x, y) \in \operatorname{dom}(g) \times \mathbb{R}: y \geq g(x)\}
$$

Here the effective domain of $g$ is given by

$$
\operatorname{dom}(g):=\left\{x \in \mathbb{R}^{n}: g(x)<\infty\right\}
$$

A vector $\zeta \in \mathbb{R}^{n}$ is said to be a proximal subgradient (or P-subgradient) of $g$ at a point $x \in \operatorname{dom}(g)$ provided that

$$
(\zeta,-1) \in N_{\mathrm{epi}(g)}^{P}(x, g(x))
$$

The set of all such vectors is called the P-subdifferential of $g$ at $x$, denoted $\partial_{P} g(x)$. A vector $\zeta \in \partial_{P} g(x)$ iff there exists $\sigma>0$ such that

$$
g(y)-g(x)+\sigma\|y-x\|^{2} \geq\langle\zeta, y-x\rangle
$$

for all $y$ near $x$; this is known as the proximal subgradient inequality. One can show that $\partial_{P} g(x) \neq \phi$ for a dense subset of $\operatorname{dom}(g)$, where we adopt the convention that $\partial_{P} g(x)=\phi$ when $x \notin \operatorname{dom}(g)$.

The limiting normal cone (or L-normal cone) to $Z$ at $x \in Z$ is defined to be the set

$$
N_{Z}^{L}(x):=\left\{\zeta \in \mathbb{R}^{n}: \zeta_{i} \rightarrow \zeta, \zeta_{i} \in N_{Z}^{P}\left(x_{i}\right), x_{i} \rightarrow x\right\}
$$

One has $N_{Z}^{L}(x) \neq\{0\}$ for every $x \in \operatorname{bdry}(Z)$. The L-normal cone leads to a corresponding L-subdifferential set for $g$ :

$$
\begin{aligned}
\partial_{L} g(x) & :=\left\{\zeta \in \mathbb{R}^{n}:(\zeta,-1) \in N_{\mathrm{epi}(g)}^{L}(x, g(x)\}\right. \\
& =\left\{\lim \zeta_{i}: \zeta_{i} \in \partial_{P} g\left(x_{i}\right), x_{i} \rightarrow x, g\left(x_{i}\right) \rightarrow g(x)\right\},
\end{aligned}
$$

the members of which are called L-subgradients.

The Clarke normal cone (or C-normal cone) to $Z$ at $x \in Z$ is defined by

$$
N_{Z}^{C}(x):=\overline{\operatorname{co}}\left[N_{S}^{L}(x)\right]
$$


and, with $g$ assumed now to be Lipschitz near $x$, the corresponding subdifferential for $g$ at $x$ is the compact set

$$
\partial_{C} g(x):=\left\{\zeta \in \mathbb{R}^{n}:(\zeta,-1) \in N_{\mathrm{epi}(g)}^{C}(x, g(x)\}=\operatorname{co}\left\{\partial_{L} g(x)\right\} .\right.
$$

We will make use of the fact that

$$
-\partial_{C}(-g)(x)=\partial_{C} g(x)
$$

For a closed set $Z$ and locally Lipschitz function $g$, one has the containments

$$
N_{Z}^{P}(x) \subset N_{Z}^{L}(x) \subset N_{Z}^{C}(x) \quad \forall x \in Z
$$

and

$$
\partial_{P} g(x) \subset \partial_{L} g(x) \subset \partial_{C} g(x) \quad \forall x \in \mathbb{R}^{n}
$$

The Clarke tangent cone (or $C$-tangent cone) to $Z$ at $x \in S$ is the cone dual to $N_{Z}^{C}(x)$; that is

$$
T_{Z}^{C}(x):=\left\{v \in \mathbb{R}^{n}:\langle v, \zeta\rangle \leq 0 \forall \zeta \in N_{Z}^{C}(x)\right\} .
$$

\subsubsection{Quadratic inf-convolutions and semiconcavity}

Let $g: \mathbb{R}^{n} \rightarrow(-\infty, \infty]$ be an extended real valued and lower semicontinuous function which is globally bounded below. Given $\lambda>0$, the quadratic inf-convolution of $g$ is the function

$$
g_{\lambda}(x):=\inf _{y \in \mathbb{R}^{n}}\left\{g(y)+\lambda\|x-y\|^{2}\right\} .
$$

Obviously $g_{\lambda}$ is majorized by $g$. It can be shown that $g_{\lambda}$ is locally Lipschitz on $\mathbb{R}^{n}$. Also, if $x \in \mathbb{R}^{n}$ is such that $\partial_{P} g_{\lambda}(x)$ is nonempty, then there exists a point $\bar{y} \in \mathbb{R}^{n}$ such that the infimum in (6) is uniquely attained at $\bar{y}$, and $\partial_{P} g_{\lambda}(x)$ is the singleton

$$
g_{\lambda}^{\prime}(x)=2 \lambda(x-\bar{y}) \in \partial_{P} g(\bar{y})
$$

where $g_{\lambda}^{\prime}(x)$ denotes the Fréchet derivative. The above facts are all verified in [17]; the specific fact that we shall require below is the containment $\partial_{P} g_{\lambda}(x) \subset \partial_{P} g(\bar{y})$.

Now suppose that $g$ is continuous. Fix $x_{0} \in \mathbb{R}^{n}$, and for a given $\varepsilon_{0}>0$, denote

$$
Q_{0}:=\max _{x \in \bar{B}_{n}\left(x_{0} ; \varepsilon_{0}\right)} g(x)
$$

Then for every $\lambda>0$ one has

$$
g_{\lambda}(x) \leq g(x) \leq Q_{0} \quad \forall x \in \bar{B}_{n}\left(x_{0} ; \varepsilon_{0}\right) .
$$

Let $x \in \bar{B}_{n}\left(x_{0} ; \varepsilon_{0}\right)$, and consider $y \in \mathbb{R}^{n}$ such that

$$
g(y)+\lambda\|y-x\|^{2} \leq Q_{0}+1 ;
$$

it is clear that such points $y$ exist. Denote the global lower bound on $g$ by $c$. Then

$$
\|y-x\| \leq \sqrt{\frac{Q_{0}+1-c}{\lambda}}:=w_{0}(\lambda) .
$$


We conclude that for every $x \in \bar{B}_{n}\left(x_{0} ; \varepsilon_{0}\right)$,

$$
g_{\lambda}(x)=\min _{y \in \bar{B}_{n}\left(x_{0} ; \varepsilon_{0}\right)+w_{0}(\lambda) \bar{B}_{n}}\left\{g(y)+\lambda\|y-x\|^{2}\right\},
$$

or

$$
-g_{\lambda}(x)=\max _{y \in \bar{B}_{n}\left(x_{0} ; \varepsilon_{0}\right)+w_{0}(\lambda) \bar{B}_{n}}\left\{-g(y)-\lambda\|x-y\|^{2}\right\} .
$$

Let us now also assume that $g$ itself is locally Lipschitz. Then (7) amounts to $-g_{\lambda}$ being $\lambda$-lower- $C^{2}$ on $B_{n}\left(x_{0} ; \varepsilon_{0}\right)$, in the terminology of Rockafellar, who studied this class of functions in [35]. Furthermore, as is shown in Clarke et al. [18], this property implies that one has the uniform version of the proximal subgradient inequality given by

$$
-g_{\lambda}(y)+g_{\lambda}(x)+\lambda\|y-x\|^{2} \geq\langle\zeta, y-x\rangle \quad \forall \zeta \in \partial_{P}\left(-g_{\lambda}\right)(x), \quad \forall x, y \in B_{n}\left(x_{0} ; \varepsilon_{0}\right) .
$$

Since $\varepsilon_{0}$ was arbitrary, equation (8) holds for all $x$ and $y$ in $\mathbb{R}^{n}$, It follows that the function $h_{\lambda}(x):=-g_{\lambda}(x)+$ $\lambda\|x\|^{2}$ is convex on $\mathbb{R}^{n}$. The argument used in showing this involves applying the "semi-smooth" proximal sum rule (Prop. 1.2.11 in [17]) to $h_{\lambda}$ in order to deduce that for each $x \in \mathbb{R}^{n}$,

$$
h_{\lambda}(y)-h_{\lambda}(x) \geq\langle\eta, y-x\rangle \quad \forall \eta \in \partial_{P} h_{\lambda}(x), \quad \forall y \in \mathbb{R}^{n},
$$

which characterizes convexity of $h_{\lambda}$. We go on to say that $g_{\lambda}$ is semiconcave on $\mathbb{R}^{n}$, since $g_{\lambda}$ is the sum of the concave function $-h_{\lambda}$ and a quadratic. A useful fact that ensues is

$$
\partial_{P}\left(-g_{\lambda}\right)(x)=\partial_{L}\left(-g_{\lambda}\right)(x)=\partial_{C}\left(-g_{\lambda}\right)(x) \quad \forall x \in \mathbb{R}^{n} .
$$

\subsection{Extended dynamics}

The function $f$ featured in the dynamics (1) has only been defined for state values $x \in S$, where $S$ is the (weakly invariant) state constraint set in the problem under study. In many problems arising from economics and engineering, for example, such a restricted definition is quite sensible, since the dynamics might not make sense or break down when $x \notin S$. On the other hand, it will be convenient for us to now extend $f$ from $S \times U$ to $\mathbb{R}^{n} \times U$ in a suitable way.

Let $f_{i}$ denote the $i^{\text {th }}$ component function of $f, i=1,2, \ldots, n$. For each fixed $u \in U$, define a function $x \rightarrow \hat{f}_{i}(x, u)$ on $\mathbb{R}^{n}$ as follows.

$$
\hat{f}_{i}(x, u)=\min _{y \in S}\left\{f_{i}(y, u)+K\|y-x\|\right\}
$$

It is not difficult to show that $x \rightarrow \hat{f}_{i}(x, u)$ agrees with $f_{i}(x, u)$ on $S$, and is globally Lipschitz of rank $K$. We extend $f$ by componentwise setting $f_{i}(x, u)=\hat{f}_{i}(x, u)$ for every $(x, u) \in \mathbb{R}^{n} \times U$. The resulting function $f: \mathbb{R}^{n} \times U \rightarrow \mathbb{R}^{n}$ is continuous on $\mathbb{R}^{n} \times U$ and is globally Lipschitz of rank $K$ in the state variable $x$, uniformly for $u \in U$; that is,

$$
\|f(x, u)-f(y, u)\| \leq K\|x-y\|,
$$

whenever $(x, u)$ and $(y, u)$ are in $\mathbb{R}^{n} \times U$. It follows that for any $(\tau, \alpha) \in \mathbb{R} \times \mathbb{R}^{n}$ and any control function $u(\cdot)$, there exists a unique trajectory $x(t)=x(t ; \tau, \alpha, u(\cdot))$ satisfying $x(\tau)=\alpha$ and defined on $[\tau, \infty)$. (Note that the global Lipschitz condition on the extended dynamics implies the linear growth condition needed for the global extendability of solutions.) Lastly, we mention that the velocity sets $f(x, U)$ need not be convex for $x \notin S$, but this poses no difficulty in the sequel. 


\subsection{Discretized solutions}

We shall present a discretized solution concept for (1) under a (generally discontinuous) control feedback $k(t, x)$, modeled by

$$
\dot{x}=f(x, k(t, x)) \text {. }
$$

Let an initial phase $(\tau, \alpha) \in(-\infty, T] \times \mathbb{R}^{n}$ be specified. Then given a partition

$$
\pi=\left\{\tau=t_{0}, t_{1}, \ldots, t_{N_{\pi}}=T\right\}
$$

of $[\tau, T]$, the associated $\pi$-trajectory $x_{\pi}$ on $[\tau, T]$ with $x_{\pi}(\tau)=\alpha$ is the curve satisfying interval-by-interval dynamics as follows: set $x_{0}=\alpha$. Then on the interval $\left[t_{0}, t_{1}\right], x_{\pi}$ is the classical solution of the differential equation

$$
\dot{x}_{\pi}(t)=f\left(x_{\pi}(t), k\left(t_{0}, x_{0}\right)\right), \quad x_{\pi}\left(t_{0}\right)=x_{0}, \quad t \in\left(t_{0}, t_{1}\right) .
$$

We then set $x_{1}:=x_{\pi}\left(t_{1}\right)$, and restart the system on the next interval as follows:

$$
\dot{x}_{\pi}(t)=f\left(x_{\pi}(t), k\left(t_{1}, x_{1}\right)\right), \quad x_{\pi}\left(t_{1}\right)=x_{1}, \quad t \in\left(t_{1}, t_{2}\right) .
$$

The process is continued in this manner through the last interval $\left[t_{N_{\pi}-1}, t_{N_{\pi}}\right]$. Note that $x_{\pi}$ is the unique solution on $[\tau, T]$ of the differential equation $\dot{x}=f(x, u)$ satisfying $x(\tau)=\alpha$, with a certain piecewise constant control function $u$ determined by the control feedback $k(t, x)$. This solution procedure, which involves discretizing the control feedback control law $k(t, x)$ via a procedure sometimes referred to as closed loop system sampling, is the same as the "step-by-step" solution concept employed by Krasovskiǐ and Subbotin [28] in differential game theory, and by Clarke et al. in in control feedback stabilization [11].

\section{INNER APPROXIMATIONS AND STATE CONSTRAINED TRAJECTORY TRACKING}

As was mentioned in the introduction, we shall require a result on state constrained trajectory tracking. Lemma 3.9 below generalizes a result due to Soner [37], where $S$ was assumed to be compact and have $C^{2}$ smooth boundary - conditions which are less general than those we shall impose, which include no smoothness. We refer the reader to Bardi and Capuzzo-Dolcetta [4] for an exposition of Soner's original result as well as additional references, and for some ramifications regarding continuity of the value function in state constrained infinite horizon control problems. We also point the reader to the work of Forcellini and Rampazzo [19], Frankowska and Rampazzo [20] as well as Rifford [31] for results allowing nonsmoothness of the boundary of $S$. Unlike the above-mentioned works, however, the tracking lemma we derive below will also apply to inner approximations of $S$ in a required uniform manner.

We denote the lower Hamiltonian $h: \mathbb{R}^{n} \times \mathbb{R}^{n} \rightarrow \mathbb{R}$ by

$$
h(x, p):=\min _{u \in U}\langle f(x, u), p\rangle .
$$

The following geometric hypotheses on the state constraint set $S$ are posited:

(S1) $S$ is compact and wedged at each $x \in \operatorname{bdry}(S)$. This means that at each boundary point $x$ one has that $N_{S}^{C}(x)$ is pointed; that is, $N_{S}^{C}(x) \cap\left\{-N_{S}^{C}(x)\right\}=\{0\}$.

(S2) The following "strict inwardness" condition holds:

$$
h(x, \zeta)<0 \quad \forall 0 \neq \zeta \in N_{S}^{C}(x), \quad \forall x \in \operatorname{bdry}(S) .
$$




\section{Remark 3.1.}

(a) Condition (S1) is equivalent to $T_{S}^{C}(x)$ having nonempty interior for each $x \in \operatorname{bdry}(S)$. A vector $v$ being interior to $T_{S}^{C}(x)$ is equivalent to the existence of $\varepsilon>0$ such that

$$
y+W(v ; \varepsilon) \subset S \quad \forall y \in\left\{x+\varepsilon B_{n}\right\} \cap S,
$$

where $W(v ; \varepsilon)$ denotes the wedge of axis $v$ and radius $\varepsilon$; that is

$$
W(v ; \varepsilon):=\left\{t w: t \in[0, \varepsilon], w \in v+\varepsilon B_{n}\right\} .
$$

(b) Wedgedness of $S$ at $x \in \operatorname{bdry}(S)$ is also referred to as epi-Lipschitzness in the literature, since it amounts to $S$ being locally linearly homeomorphic to the epigraph of a Lipschitz function; see Rockafellar [34] and Clarke [8]. When $S$ is wedged, the multifunction $x \rightarrow N_{S}^{C}(x) \cap \Sigma_{n}$ has closed graph on $S$.

Also one has

$$
S=\operatorname{cl}[\operatorname{int}(S)]
$$

and

$$
N_{S}^{C}(x)=-N_{\hat{S}}^{C}(x) \quad \forall x \in \operatorname{bdry}(S)=\operatorname{bdry}(\hat{S}) ;
$$

see e.g. [16]. An analytical version of this fact is that for a function $g$ which is Lipschitz near a point $x$, one has

$$
\partial_{C} g(x)=-\partial_{C}(-g)(x)
$$

(c) Weak invariance of $S$ is equivalent to the condition

$$
h(x, \zeta) \leq 0 \quad \forall \zeta \in N_{S}^{P}(x), \quad \forall x \in \operatorname{bdry}(S) .
$$

Hence condition (S2) is (only) sufficient for weak invariance. The above proximal characterization of weak invariance follows from one in Chapter 4 of [17], which is stated for dynamics given in terms of a differential inclusion $\dot{x} \in F(x)$, with the multifunction $F$ being globally defined and satisfying certain standard hypotheses, including convexity of $F(x)$ for every $x \in \mathbb{R}^{n}$. The characterization in [17] becomes applicable in our setting by taking $F(x):=\operatorname{cof}(x, U)$, because $F(x)=f(x, U)$ when $x \in S$, and every trajectory of the differential inclusion that remains in $S$ is also a trajectory of the control system (1), by Filippov's lemma.

(d) The fact that the multifunction $x \rightarrow N_{S}^{C}(x) \cap \Sigma_{n}$ has closed graph when (S1) holds implies that the same is true of the multifunction $x \rightarrow \operatorname{co}\left\{N_{S}^{C}(x) \cap \Sigma_{n}\right\}$; this fact is a straightforward exercise involving Carathéodory's theorem. In view of $(\mathrm{S} 1)$, for $x \in \operatorname{bdry}(S)$, one has $0 \notin \operatorname{co}\left\{N_{S}^{C}(x) \cap \Sigma_{n}\right\}$. It follows that there exists $\mu>0$ such that

$$
h(x, \zeta)<-\mu \quad \forall \zeta \in \operatorname{co}\left\{N_{S}^{C}(x) \cap \Sigma_{n}\right\}, \quad \forall x \in \operatorname{bdry}(S),
$$

which we can write as

$$
\max _{\zeta \in \operatorname{co}\left\{N_{S}^{G}(x) \cap \Sigma_{n}\right\}} \min _{u \in U}\langle f(x, u), \zeta\rangle<-\mu \quad \forall x \in \operatorname{bdry}(S) .
$$

The minimax theorem (which is applicable by virtue of hypothesis (F2)) then implies

$$
\min _{u \in U} \max _{\zeta \in \operatorname{co}\left\{N_{S}^{C}(x) \cap \Sigma_{n}\right\}}\langle f(x, u), \zeta\rangle<-\mu \quad \forall x \in \operatorname{bdry}(S) .
$$


This is easily seen to be equivalent to the existence, for each $x \in \operatorname{bdry}(S)$, of $u_{x} \in U$ such that

$$
\left\langle f\left(x, u_{x}\right), \zeta\right\rangle \leq-\mu \quad \forall \zeta \in \operatorname{co}\left\{N_{S}^{C}(x) \cap \Sigma_{n}\right\},
$$

which is in turn equivalent to

$$
\left\langle f\left(x, u_{x}\right), \zeta\right\rangle \leq-\mu \quad \forall \zeta \in N_{S}^{C}(x) \cap \Sigma_{n} .
$$

It is convenient to rephrase these statements in terms of the $C$-tangent cone as follows:

$$
f\left(x, u_{x}\right)+\mu B_{n} \subset T_{S}^{C}(x) .
$$

(The equivalence of (24) and (25) follows from the polarity of $N_{S}^{C}(x)$ and $T_{S}^{C}(x)$.)

\subsection{Inner approximations}

Given $r \geq 0$, we denote the $r$-inner approximation of $S$ by

$$
S_{r}:=\left\{x \in \mathbb{R}^{n}: d_{\hat{S}}(x) \geq r\right\} .
$$

Note that $S_{0}=S$. Geometric properties of inner approximations of this type were studied in Clarke et al. [16].

Given $x \in S$, let $r(x):=d_{\hat{S}}(x)$. We denote

$$
N(x):=N_{S_{r(x)}}^{C}(x) \cap \Sigma_{n}
$$

and

$$
T(x):=T_{S_{r(x)}}^{C}(x)=\left\{v \in \mathbb{R}^{n}:\langle v, \zeta\rangle \leq 0 \forall \zeta \in N(x)\right\} .
$$

We require the following preliminary lemma concerning pointedness.

Lemma 3.2. Suppose that for some fixed positive integer $m$ one has

$$
\zeta=\lim _{k \rightarrow \infty} \sum_{j=1}^{m} \alpha_{j}^{k} \eta_{j}^{k},
$$

where $\alpha_{j}^{k} \geq 0$ for each $j$ and $k$. Let $\lim _{k \rightarrow \infty} \eta_{j}^{k}=\eta_{j}$ for each $j$, where $0 \neq \eta_{j} \in C$, and where $C \subset \mathbb{R}^{n}$ is a closed, convex and pointed cone. Then there exist scalars $\alpha_{j} \geq 0$ such that

$$
\zeta=\sum_{j=1}^{m} \alpha_{j} \eta_{j}
$$

Proof. The properties of $C$ imply the existence of $p \in \mathbb{R}^{n}$ such that $\left\langle p, \eta_{j}\right\rangle>0$ for each $j$. It then follows from (26) that the sequence $\alpha_{j}^{k}$ is bounded for each $j$, for otherwise $\langle p, \zeta\rangle$ would not be finite, which is impossible. The proof of the lemma is now easily completed by taking subsequences.

The next lemma provides useful information about the behavior of $N(\cdot)$ and $T(\cdot)$ on a compact "inner tube" of $S$, under the assumption of wedgedness. For $r \geq 0$ we denote such an inner tube by

$$
Q(S ; r):=S \backslash \operatorname{int}\left(S_{r}\right) .
$$


Lemma 3.3. Let (S1) hold. Then there exists $r_{0}>0$ such that $S_{r}$ is nonempty and wedged for all $r \in\left[0, r_{0}\right]$. Furthermore, for any such $r_{0}$, one has

(a) the multifunction $N(\cdot)$ has closed graph on $Q\left(S ; r_{0}\right)$, which is equivalent to

(b) the multifunction $T(\cdot)$ is lower semicontinuous on $Q\left(S ; r_{0}\right)$.

The assertation regarding wedgedness of inner approximations is proven in [16], and is therefore omitted. Part (a) asserts that the set

$$
\left\{(x, \zeta): x \in Q\left(S ; r_{0}\right), \zeta \in N(x)\right\}
$$

is closed, while part (b) asserts that for any $x \in Q\left(S ; r_{0}\right)$, given $v \in T(x)$ and $\varepsilon>0$, there exists $\delta>0$ such that

$$
y \in Q\left(S ; r_{0}\right) \cap\left\{x+\delta B_{n}\right\} \Longrightarrow v \in T(y)+\varepsilon B_{n} .
$$

The proof that (a) and (b) are equivalent is essentially the same as that of Proposition 3.6.8 in [17], and is therefore omitted as well.

Proof of part (a). Let $\left(x_{i}, \zeta_{i}\right) \rightarrow(x, \zeta)$ as $i \rightarrow \infty$, where $x_{i} \in Q\left(S ; r_{0}\right)$ and $\zeta_{i} \in N\left(x_{i}\right)$ for each $i$. Then $x \in Q\left(S ; r_{0}\right)$ and we are to show that $\zeta \in N(x)$. There are two cases to consider.

Case 1: $x \in \operatorname{int}(S)$.

For large $i$ we have

$$
\zeta_{i} \in N_{S_{r\left(x_{i}\right)}^{C}}^{C}\left(x_{i}\right) \cap \Sigma_{n}
$$

by assumption. We temporarily fix such an $i$. In view of (17) and the definition of the $C$-normal cone, for every $\varepsilon>0$ we have

$$
-\zeta_{i} \in \overline{\mathrm{CO}}\left\{N_{\widehat{S_{r\left(x_{i}\right)}}}^{P}(y): y \in \operatorname{bdry}\left(S_{r\left(x_{i}\right)}\right),\left\|y-x_{i}\right\|<\varepsilon\right\} .
$$

Furthermore, if $\eta \in N_{\widehat{S_{r\left(x_{i}\right)}}}^{P}(y)$, one has $\eta \in N_{\hat{S}}^{P}(w)$, where $\operatorname{proj}_{\hat{S}}(y)=\{w\}$; see e.g. Lemma 3.3 of [18]. It is not difficult to verify that then we also have $y \in \operatorname{proj}_{S_{r\left(x_{i}\right)}}(w)$. It follows from $(28)$ and Carathéodory's theorem that for each $i$ one has

$$
\zeta_{i}=\lim _{k \rightarrow \infty} \sum_{j=1}^{n+1} \alpha_{j}^{i, k} \frac{w_{j}^{i, k}-y_{j}^{i, k}}{\left\|w_{j}^{i, k}-y_{j}^{i, k}\right\|}
$$

where $y_{j}^{i, k} \in \operatorname{proj}_{S_{r\left(x_{i}\right)}}\left(w_{j}^{i, k}\right), w_{j}^{i, k} \in \operatorname{bdry}(S),\left\|y_{j}^{i, k}-x_{i}\right\|<\frac{1}{k}$, and $\alpha_{j}^{i, k} \geq 0$. (Note also that $\left\|w_{j}^{i, k}-y_{j}^{i, k}\right\|=$ $r\left(x_{i}\right)=d_{\hat{S}}\left(x_{i}\right)$.) For each $j$, upon taking subsequences (not relabeled),

$$
\frac{w_{j}^{i, k}-y_{j}^{i, k}}{\left\|w_{j}^{i, k}-y_{j}^{i, k}\right\|} \rightarrow \frac{w_{j}^{i}-x_{i}}{\left\|w_{j}^{i}-x_{i}\right\|}=: \eta_{j}^{i}
$$

as $k \rightarrow \infty, w_{j}^{i} \in \operatorname{bdry}(S)$, and $x_{i} \in \operatorname{proj}_{S_{r\left(x_{i}\right)}}\left(w_{j}^{i}\right)$; that is, $\left\|w_{j}^{i}-x_{i}\right\|=r\left(x_{i}\right)=d_{\hat{S}}\left(x_{i}\right)$. It follows that for each $j$

$$
\eta_{j}^{i} \in N_{S_{r\left(x_{i}\right)}}^{P}\left(x_{i}\right) \cap \Sigma_{n} \subset N_{S_{r\left(x_{i}\right)}}^{C}\left(x_{i}\right) \cap \Sigma_{n} .
$$


Then Lemma 3.2 yields the existence of nonnegative scalars $\alpha_{j}^{i}$ such that

$$
\zeta_{i}=\sum_{j=1}^{n+1} \alpha_{j}^{i} \eta_{j}^{i}
$$

Now consider the convergence $\zeta_{i} \rightarrow \zeta$ as $i \rightarrow \infty$. For each $j$, along a subsequence (not relabeled), we have

$$
\lim _{i \rightarrow \infty} \eta_{j}^{i} \rightarrow \eta_{j}=\frac{w_{j}-x}{\left\|w_{j}-x\right\|}
$$

where $x \in \operatorname{proj}_{S_{r(x)}}\left(w_{j}\right)$ and $w_{j} \in \operatorname{bdry}(S)$. Upon noting that each $\eta_{j} \in N_{S_{r(x)}}^{P}(x)=N_{S_{r(x)}}^{C}(x)$, another application of Lemma 3.2 implies

$$
\zeta=\sum_{j=1}^{n+1} \alpha_{j} \eta_{j}
$$

for nonnegative scalars $\alpha_{j}$. We conclude that $\zeta \in N_{S_{r(x)}}^{C}(x)$, as required.

Case 2: $x \in \operatorname{bdry}(S)$.

If there exists an infinite subsequence of $x_{i}$ contained in $\operatorname{bdry}(S)$, then the conclusion follows from graph closedness of the multifunction $x \rightarrow N_{S}^{C}(x) \cap \Sigma_{n}$ on $S$. So let us assume $x_{i} \in \operatorname{int}(S)$ for each $i$, with $x_{i} \rightarrow x \in$ $\operatorname{bdry}(S)$. Formula (32) again holds; that is,

$$
\zeta_{i}=\sum_{j=1}^{n+1} \alpha_{j}^{i} \eta_{j}^{i} ; \quad \eta_{j}^{i}=\frac{w_{j}^{i}-x_{i}}{\left\|w_{j}^{i}-x_{i}\right\|}
$$

where each $\alpha_{j}^{i} \geq 0, w_{j}^{i} \in \operatorname{bdry}(S)$, and $\left\|x_{i}-w_{j}^{i}\right\|=r\left(x_{i}\right)=d_{\hat{S}}\left(x_{i}\right)$. Then $w_{j}^{i} \in \operatorname{proj}_{\hat{S}}\left(x_{i}\right)$ and consequently,

$$
-\eta_{j}^{i}=\frac{x_{i}-w_{j}^{i}}{\left\|x_{i}-w_{j}^{i}\right\|} \in N_{\hat{S}}^{P}\left(w_{j}^{i}\right) \cap \Sigma_{n} \subset N_{\hat{S}}^{C}\left(w_{j}^{i}\right) \cap \Sigma_{n}=\left\{-N_{S}^{C}\left(w_{j}^{i}\right)\right\} \cap \Sigma_{n} .
$$

Since $w_{j}^{i} \rightarrow x$ as $i \rightarrow \infty$ for each $j$, the graph closedness of the multifunction $x \rightarrow N_{S}^{C}(x) \cap \Sigma_{n}$ on $S$ implies $\eta_{j}^{i} \rightarrow \eta_{j} \in N_{S}^{C}(x) \cap \Sigma_{n}$ for each $j$. Then (32) and Lemma 3.2 again imply (34), and therefore $\zeta \in N_{S}^{C}(x)$, as required.

In the sequel, we fix a scalar $\mu$ as in (20). From the preceding lemma, the continuity of the dynamics $f$, part (c) of Remark 3.1, as well as the arguments employed in part (d) of that remark, we obtain the following conclusions.

Corollary 3.4. Under hypotheses (S1) and (S2), $r_{0}>0$ can be taken small enough to guarantee that for every $r \in\left[0, r_{0}\right]$, the inner approximation $S_{r}$ is wedged and satisfies

$$
h(x, \zeta)<-\frac{\mu}{2} \quad \forall \zeta \in \operatorname{co}\{N(x)\}, \quad \forall x \in Q\left(S ; r_{0}\right) .
$$

In particular, for every such $r, S_{r}$ is weakly invariant. 
Note that, analogously to the way we arrived at (24) from (20) via the minimax theorem, the preceding corollary implies that for each $x \in Q\left(S ; r_{0}\right)$, there exists $u_{x} \in U$ such that

$$
\left\langle f\left(x, u_{x}\right), \zeta\right\rangle<-\frac{\mu}{2} \quad \forall \zeta \in N(x),
$$

which is in turn equivalent to

$$
f\left(x, u_{x}\right)+\frac{\mu}{2} B_{n} \subset T(x) .
$$

- From this point on, we shall assume that $r_{0}$ is as in the preceding corollary, and take $u_{x}$ (and $\mu$ ) to be as in (37).

We will require the following facts concerning a certain continuous selection of $f(x, U)$ as $x$ varies in an inner tube.

Lemma 3.5. Let (S1) and (S2) hold. Then there exists a Lipschitz function $v: Q\left(S ; r_{0}\right) \rightarrow \mathbb{R}^{n}$ such that

$$
v(x) \in f(x, U) \quad \forall x \in Q\left(S ; r_{0}\right)
$$

and

$$
v(x)+\frac{\mu}{3} B_{n} \subset T(x) \quad \forall x \in Q\left(S ; r_{0}\right) .
$$

Furthermore, (40) is equivalent to

$$
\langle v(x), \zeta\rangle<-\frac{\mu}{3} \quad \forall \zeta \in N(x), \quad \forall x \in Q\left(S ; r_{0}\right) .
$$

Proof. In view of (25), the continuity of $f$ and the lower semicontinuity property of $T(\cdot)$ provided in Lemma 3.3(b), one has that for each $x \in Q\left(S ; r_{0}\right)$ there exists $u_{x} \in U$, and an open neighborhood $A_{x}$ of $x$, such that

$$
f\left(z, u_{x}\right)+\frac{\mu}{3} B_{n} \subset T(x) \quad \forall z \in A_{x} .
$$

The family $\left\{A_{x}\right\}$ forms an open covering of the compact set $Q\left(S ; r_{0}\right)$. Let $\left\{A_{x_{i}}\right\}$ be a finite subcover, and associate with it a Lipschitz partition of unity $\left\{p_{i}(\cdot)\right\}$. We define

$$
v(x):=\Sigma_{i} p_{i}(x) f\left(x, u_{x_{i}}\right) .
$$

Then (F2) (convexity of $f(x, U)$ for $x \in S$ ) and convexity of $T(x)$ readily yield (39) and (40). We leave the straightforward proof of the "furthermore" part to the reader.

The next lemma provides for uniformity of the radii of wedges with axes $v(x)$ or $-v(x)$.

Lemma 3.6. Let (S1) and (S2) hold. Then there exists $\tilde{\varepsilon}>0$ such that

$$
y+W(v(x) ; \tilde{\varepsilon}) \subset S_{r(x)} \quad \forall y \in\left\{x+\tilde{\varepsilon} B_{n}\right\} \cap S_{r(x)}, \quad \forall x \in Q\left(S ; r_{0}\right)
$$

and

$$
y+W(-v(x) ; \tilde{\varepsilon}) \subset \widehat{S_{r(x)}} \quad \forall y \in\left\{x+\tilde{\varepsilon} B_{n}\right\} \cap \widehat{S_{r(x)}}, \quad \forall x \in Q\left(S ; r_{0}\right)
$$


Proof. We shall proceed by way of contradiction. First assume that no $\tilde{\varepsilon}>0$ satisfying (44) exists. Then there exist sequences $x_{j} \in Q\left(S ; r_{0}\right), \varepsilon_{j} \downarrow 0, t_{j} \in\left(0, \varepsilon_{j}\right], w_{j} \in B_{n}$ and $y_{j} \in\left\{x_{j}+\varepsilon_{j} B_{n}\right\} \cap S_{r\left(x_{j}\right)}$ such that

$$
y_{j}+t_{j}\left(v\left(x_{j}\right)+\varepsilon_{j} w_{j}\right) \notin S_{r\left(x_{j}\right)},
$$

or equivalently,

$$
d_{S_{r\left(x_{j}\right)}}\left(y_{j}+t_{j}\left(v\left(x_{j}\right)+\varepsilon_{j} w_{j}\right)\right)-d_{S_{r\left(x_{j}\right)}}\left(y_{j}\right)>0 .
$$

The (unidirectional) proximal mean value theorem (see [17]) implies that for each $j=1,2, \ldots$, there exist

$$
z_{j} \in\left[y_{j}, y_{j}+t_{j}\left(v\left(x_{j}\right)+\varepsilon_{j} w_{j}\right)\right]+\frac{1}{j} B_{n}
$$

and

$$
0 \neq \eta_{j} \in \partial_{P} d_{S_{r\left(x_{j}\right)}}\left(z_{j}\right) \subset \partial_{C} d_{S_{r\left(x_{j}\right)}}\left(z_{j}\right) \subset N_{S_{r\left(x_{j}\right)}}^{C}\left(z_{j}\right)
$$

such that

$$
\left\langle v\left(x_{j}\right)+\varepsilon_{j} w_{j}, \frac{\eta_{j}}{\left\|\eta_{j}\right\|}\right\rangle>0 .
$$

Upon taking subsequences (not relabeled), we obtain $z_{j} \rightarrow x \in Q\left(S ; r_{0}\right)$ and $\frac{\eta_{j}}{\left\|\eta_{j}\right\|} \rightarrow \zeta \in N(x)$ (since the multifunction $N(\cdot)$ has closed graph, by Lem. 3.3). Then the continuity of $v(\cdot)$ implies $\langle v(x), \zeta\rangle \geq 0$, which contradicts (41).

Bearing (17) in mind, it is clear that the same contradiction would occur if no $\tilde{\varepsilon}>0$ satisfying (45) existed, which completes the proof.

\subsection{Signed distance function}

For a closed set $Z \subset \mathbb{R}^{n}$, we define the signed distance function as

$$
\Delta_{Z}(x):=d_{Z}(x)-d_{\hat{Z}}(x) .
$$

See Hiriart-Urruty [22] for early results concerning this function. It is not difficult to see that $\Delta_{Z}$ is Lipschitz of rank 2 , and a standard $C$-calculus argument shows that

$$
\partial_{C} \Delta_{Z}(x) \subset N_{Z}^{C}(x) \cap\left\{2 \bar{B}_{n}\right\} \quad \forall x \in \operatorname{bdry}(Z) .
$$

We shall also require the following result.

Lemma 3.7. Let $Z \subset \mathbb{R}^{n}$ be a closed set which is wedged at $x \in \operatorname{bdry}(Z)$, and suppose $v \in \mathbb{R}^{n}$ and $\varepsilon>0$ are such that

$$
y+W(v ; \varepsilon) \subset Z \quad \forall y \in\left\{x+\varepsilon B_{n}\right\} \cap Z,
$$

and

$$
y+W(-v ; \varepsilon) \subset \hat{Z} \quad \forall y \in\left\{x+\varepsilon B_{n}\right\} \cap \hat{Z} .
$$

Then

$$
\langle v, \zeta\rangle \leq-\varepsilon \quad \forall \zeta \in \partial_{C} \Delta_{Z}(x) .
$$


Proof. Since the boundary of $Z$ is locally linearly homeomorphic to the graph of a Lipschitz function (recall Rem. 3.1(b)), for some $\gamma>0$ one has

$$
\operatorname{meas}\left[\operatorname{bdry}(Z) \cap\left\{x+\gamma B_{n}\right\}\right]=0 .
$$

Therefore the generalized gradient formula in finite dimensions (see Th. 2.8.1 in [17] as well as [8]) asserts that

$$
\partial_{C} \Delta_{Z}(x)=\operatorname{co}\left\{\lim \nabla \Delta_{Z}\left(x_{i}\right): x_{i} \rightarrow x, x_{i} \notin \operatorname{bdry}(Z) \cup \Omega\right\},
$$

where $\Omega$ denotes the set of points where $\Delta_{Z}$ fails to be differentiable. In view of this formula, it is clear that the proof of the lemma will follow upon verification of the following

Claim. If $p \notin \Omega \cup \operatorname{bdry}(Z)$ is such that $\|p-x\|<\frac{\varepsilon}{2}$, then

$$
\left\langle v, \nabla \Delta_{Z}(p)\right\rangle \leq-\varepsilon
$$

We will verify the case $p \notin Z$. The case $p \in Z$ is similar and left to the reader. Then $\nabla \Delta_{Z}(p)=\nabla d_{Z}(p)$, and by a well known formula (see [8]) for the gradient of the distance function at a point exterior to $Z$, we obtain

$$
\nabla \Delta_{Z}(p)=\frac{p-s}{\|p-s\|}
$$

where $s \in \operatorname{proj}_{Z}(p)$. Note that $\|x-s\|<\varepsilon$. Then in view of (47),

$$
t \in[0, \varepsilon] \Longrightarrow s+t\left(v+\varepsilon \nabla \Delta_{Z}(p)\right) \in Z,
$$

and therefore the function

$$
g(t):=\left\|p-\left[s+t\left(v+\varepsilon \nabla \Delta_{Z}(p)\right)\right]\right\|
$$

attains a minimum over $[0, \varepsilon]$ at $t=0$. Since this is a point of differentiability of $g$, we obtain $g^{\prime}(0) \geq 0$; upon computing the derivative, this inequality readily yields (51).

From Lemmas 3.6 and 3.7 we deduce the following.

Lemma 3.8. Let (S1) and (S2) hold, and let $r_{0}, v(\cdot)$ and $\tilde{\varepsilon}$ be as above. Then for each $r \in\left[0, r_{0}\right]$ one has

$$
\|\zeta\| \geq \frac{\tilde{\varepsilon}}{\max \left\{\|v(x)\|: x \in Q\left(S ; r_{0}\right)\right\}}=: \hat{\varepsilon}>0 \quad \forall \zeta \in \partial_{C} \Delta_{S_{r}}(x), \quad \forall x \in \operatorname{bdry}\left(S_{r}\right) .
$$

\subsection{State constrained trajectory tracking}

Let us denote

$$
\hat{N}(x):=\left\{\zeta \in N_{S_{r(x)}}^{C}(x): \hat{\varepsilon} \leq\|\zeta\| \leq 2\right\}
$$

By (46) and Lemma 3.8, we have

$$
\partial_{C} \Delta_{S_{r(x)}}(x) \subset \hat{N}(x) \quad \forall x \in Q\left(S ; r_{0}\right),
$$


and therefore by (37),

$$
\left\langle f\left(x, u_{x}\right), \zeta\right\rangle<-\frac{\hat{\varepsilon} \mu}{2} \quad \forall \zeta \in \partial_{C} \Delta_{S_{r(x)}}(x), \quad \forall x \in Q\left(S ; r_{0}\right) .
$$

The next result, which we shall refer to in brief as the "tracking lemma", provides for $S_{r}$-constrained trajectory tracking, in a required uniform manner for $0 \leq r \leq r_{0}$.

Lemma 3.9. Let $(S 1, S 2)$ hold. Then there exist constants $t^{*}>0$ and $C>0$ such that for every $r \in\left[0, r_{0}\right]$, the following holds: Given any $\alpha \in S_{r}$ and any control $u(\cdot)$, there exists a control $\bar{u}(\cdot)$ such that

$$
x(t ; 0, \alpha, \bar{u}(\cdot)) \in S_{r} \quad \forall t \in\left[0, t^{*}\right]
$$

and

$$
\|x(t ; 0, \alpha, u(\cdot))-x(t ; 0, \alpha, \bar{u}(\cdot))\| \leq C \max _{t \in\left[0, t^{*}\right]} d_{S_{r}}(x(t ; 0, \alpha, u(\cdot))) \quad \forall t \in\left[0, t^{*}\right]
$$

Proof. For each $x \in Q\left(S ; r_{0}\right)$, there exists $\tilde{A}_{x}$, an open neighborhood of $x$, such that

$$
\left\langle f\left(z, u_{x}\right), \zeta\right\rangle \leq-\frac{\hat{\varepsilon} \mu}{3} \quad \forall \zeta \in \partial_{C} \Delta_{S_{r(x)}}(y), \quad \forall y, z \in \tilde{A}_{x} ;
$$

we have used here the graph closedness of $\partial_{C} \Delta_{S_{r(x)}}(\cdot)$ at $x$ as well as (54). We choose a finite subcovering $\left\{\tilde{A}_{x_{i}}\right\}$ of $Q\left(S ; r_{0}\right)$, and let $\left\{\tilde{p}_{i}\right\}$ be a subordinated Lipschitz partition of unity. By definition, for each $i, \operatorname{Supp}\left(\tilde{p}_{i}\right)$ (the support of $\tilde{p}_{i}$ ) is included in $\tilde{A}_{x_{i}}$. We denote

$$
\rho:=\min _{i} \min _{x \in \operatorname{Supp}\left(\tilde{p}_{i}\right)} d_{\mathbb{R}^{n} \backslash \tilde{A}_{x_{i}}}(x)>0 .
$$

We define a function $w: Q\left(S ; r_{0}\right) \rightarrow U$ as follows. For every $x \in Q\left(S ; r_{0}\right)$, we pick $i=i(x)$ such that $x \in \operatorname{Supp}\left(\tilde{p}_{i}\right)$, and set $w(x):=u_{x_{i}}$.

Let us now posit a temporary assumption.

$\left(\mathrm{F}^{*}\right)$ One has

$$
\sup _{(x, u) \in \mathbb{R}^{n} \times U}\|f(x, u)\|=: H<\infty .
$$

Under the fortified condition $\left(\mathrm{F}^{*}\right)$, we have the following estimate, for any control function $u(\cdot)$ :

$$
\|x(t ; 0, x, u(\cdot))-x\| \leq H t \quad \forall t \geq 0, \quad \forall x \in \mathbb{R}^{n} .
$$

Now let us fix $r \in\left[0, r_{0}\right]$. Given a control $u(\cdot)$ and initial phase $(0, x)$ with $x \in S_{r}$, we denote by $t_{x}(u(\cdot))$ the exit time of the associated trajectory from $\operatorname{int}\left(S_{r}\right)$; that is

$$
t_{x}(u(\cdot)):=\left\{\begin{array}{cl}
\infty & \text { if } x(t ; 0, x, u(\cdot)) \in \operatorname{int}\left(S_{r}\right) \forall t \geq 0 \\
\min \left\{t \geq 0: x(t ; 0, x, u(\cdot)) \in \operatorname{bdry}\left(S_{r}\right)\right\} & \text { otherwise. }
\end{array}\right.
$$

We want to determine two constants $t^{*}, k^{*}>0$, independent of $r$, such that for any $x \in S_{r}$ and any control function $u(\cdot)$, the following is true: if we set

$$
t_{0}:=\min \left\{t^{*}, t_{x}(u(\cdot))\right\}, \quad z:=x\left(t_{0} ; 0, x, u(\cdot)\right), \quad \varepsilon:=\max _{t \in\left[0, t^{*}\right]} d_{S_{r}}(x(t ; 0, x, u(\cdot))),
$$


and define

$$
\begin{cases}\bar{u}(t)=u(t) & \text { if } t_{0}=t^{*}, \\ \bar{u}(t)=u(t) \chi_{\left[0, t_{0}\right)}(t)+w(z) \chi_{\left[t_{0}, t_{0}+k^{*} \varepsilon\right]}(t)+u\left(t-k^{*} \varepsilon\right) \chi_{\left(t_{0}+k^{*} \varepsilon, \infty\right)}(t) & \text { if } t_{0}=t_{x}(u(\cdot)),\end{cases}
$$

then $x(t ; 0, x, \bar{u}(\cdot)) \in S_{r}$ for all $t \in\left[0, t^{*}\right]$. (Here $\chi(J)$ denotes the characteristic function of an interval $J$.)

For easier notation, let us write

$$
x(t):=x(t ; 0, x, u(\cdot)), \quad \bar{x}(t):=x(t ; 0, x, \bar{u}(\cdot)) .
$$

Note that if $\Delta_{S_{r}}(x) \leq-\frac{\hat{\varepsilon} \mu}{6}$, we can choose $t^{*}<\frac{\hat{\varepsilon} \mu}{6 H}$; we then obtain from (58) that $x(t) \in S_{r}$ for all $t \in\left[0, t^{*}\right]$.

Now suppose $\Delta_{S_{r}}(x)>-\frac{\hat{\varepsilon} \mu}{6}$ and $t_{0}=t_{x}(u(\cdot))<t^{*}<\frac{\rho}{2 H}$. Then $x(t)$ and $\bar{x}(t)$ remain in $\tilde{A}_{x_{i(z)}}$ on the interval $\left[0, t^{*}\right]$ by $(58)$ (and by the definition of $\rho$ ), where $z=x\left(t_{0}\right)=\bar{x}\left(t_{0}\right) \in \operatorname{bdry}\left(S_{r}\right)$. We set

$$
p:=\bar{x}\left(t_{0}+k^{*} \varepsilon\right)-z=\int_{t_{0}}^{t_{0}+k^{*} \varepsilon} f(\bar{x}(s), \bar{u}(s)) \mathrm{d} s,
$$

where $k^{*}$ such that $t_{0}+k^{*} \varepsilon<t^{*}$ is yet to be specified. Let $y \in \hat{A}_{x_{i(z)}}$ and $\zeta \in \partial_{C} \Delta_{S_{r}}(y)$. Then by the definition of $\bar{u}(\cdot)$ and by $(57)$, one has

$$
\langle\zeta, p\rangle=\left\langle\zeta, \int_{t_{0}}^{t_{0}+k^{*} \varepsilon} f\left(\bar{x}(s), w\left(x_{i}\right)\right) \mathrm{d} s\right\rangle=\int_{t_{0}}^{t_{0}+k^{*} \varepsilon}\left\langle\zeta, f\left(\bar{x}(s), w\left(x_{i}\right)\right)\right\rangle \mathrm{d} s \leq-\frac{\hat{\varepsilon} \mu}{3} k^{*} \varepsilon .
$$

A straightforward argument using the Gronwall inequality yields that for all $s>t_{0}+k^{*} \varepsilon$, one has

$$
\begin{aligned}
\left\|\bar{x}(s)-x\left(s-k^{*} \varepsilon\right)\right\| & \leq\left\|\bar{x}\left(t_{0}+k^{*} \varepsilon\right)-z\right\| e^{K\left(s-t_{0}-k^{*} \varepsilon\right)} \\
& \leq H k^{*} \varepsilon e^{K\left(s-t_{0}-k^{*} \varepsilon\right)} \text { for } s>t_{0}+k^{*} \varepsilon
\end{aligned}
$$

Now consider $t$ such that $t_{0}+k^{*} \varepsilon \leq t \leq t^{*}$. We compute

$$
\begin{aligned}
\bar{x}(t) & =z+p+\int_{t_{0}+k^{*} \varepsilon}^{t} f(\bar{x}(s), \bar{u}(s)) \mathrm{d} s \\
& =z+p+\int_{t_{0}}^{t-k^{*} \varepsilon} f\left(\bar{x}\left(s+k^{*} \varepsilon\right), u(s)\right) \mathrm{d} s \\
& =z+p+\int_{t_{0}}^{t-k^{*} \varepsilon} f(x(s), u(s)) \mathrm{d} s+\int_{t_{0}}^{t-k^{*} \varepsilon}\left[f\left(\bar{x}\left(s+k^{*} \varepsilon\right), u(s)\right)-f(x(s), u(s))\right] \mathrm{d} s \\
& =x\left(t-k^{*} \varepsilon\right)+p+\int_{t_{0}}^{t-k^{*} \varepsilon}\left[f\left(\bar{x}\left(s+k^{*} \varepsilon\right), u(s)\right)-f(x(s), u(s))\right] \mathrm{d} s .
\end{aligned}
$$

Now define the function

$$
g: s \in[0,1] \mapsto \Delta_{S_{r}}\left(x\left(t-k^{*} \varepsilon\right)+s p\right) .
$$

By the Lebourg mean value theorem (see $[8,17])$, there exist $s \in[0,1]$ and $\zeta \in \partial_{C} \Delta_{S_{r}}\left(x\left(t-k^{*} \varepsilon\right)+s p\right)$ such that

$$
g(1)-g(0) \leq\langle\zeta, p\rangle
$$


Then by (59) we obtain

$$
\Delta_{S_{r}}\left(x\left(t-k^{*} \varepsilon\right)+p\right) \leq \Delta_{S_{r}}\left(x\left(t-k^{*} \varepsilon\right)\right)-\frac{\hat{\varepsilon} \mu}{3} k^{*} \varepsilon \leq \varepsilon\left(1-\frac{\hat{\varepsilon} \mu}{3} k^{*}\right),
$$

because $\sup _{0 \leq t \leq t^{*}} d_{S_{r}}(x(t ; 0, x, u(\cdot)))=\varepsilon$. We then deduce from (60) that

$$
\begin{aligned}
\Delta_{S_{r}}(\bar{x}(t)) & =\Delta_{S_{r}}\left(x\left(t-k^{*} \varepsilon\right)+p\right)+\left[\Delta_{S_{r}}(\bar{x}(t))-\Delta_{S_{r}}\left(x\left(t-k^{*} \varepsilon\right)+p\right)\right] \\
& \leq \varepsilon\left(1-\frac{\hat{\varepsilon} \mu}{3} k^{*}\right)+2\left\|\bar{x}(t)-p-x\left(t-k^{*} \varepsilon\right)\right\| \\
& \leq \varepsilon\left(1-\frac{\hat{\varepsilon} \mu}{3} k^{*}\right)+2\left\|\int_{t_{0}}^{t-k^{*} \varepsilon}\left[f\left(\bar{x}\left(s+k^{*} \varepsilon\right), u(s)\right)-f(x(s), u(s))\right] \mathrm{d} s\right\| \\
& \leq \varepsilon\left(1-\frac{\hat{\varepsilon} \mu}{3} k^{*}\right)+2 \int_{t_{0}}^{t-k^{*} \varepsilon} K H k^{*} \varepsilon \mathrm{e}^{K\left(s-t_{0}\right)} \mathrm{d} s \\
& \leq \varepsilon\left(1-\frac{\hat{\varepsilon} \mu}{3} k^{*}\right)+2 H k^{*} \varepsilon\left[\mathrm{e}^{K\left(t-t_{0}-k^{*} \varepsilon\right)}-1\right] .
\end{aligned}
$$

Consequently, we deduce that if we choose $k^{*}:=\frac{6}{\hat{\varepsilon} \mu}$ and

$$
t^{*}:=\min \left\{\frac{\hat{\varepsilon} \mu}{6 H}, \frac{\rho}{2 H}, \frac{1}{K} \log _{e}\left(1+\frac{\hat{\varepsilon} \mu}{24 H}\right)\right\}
$$

then we have that

$$
\Delta_{S_{r}}(\bar{x}(t)) \leq-\frac{\varepsilon}{2}
$$

for all $t \in\left[t_{0}+k^{*} \varepsilon, t^{*}\right]$. On the other hand, it is clear that $\Delta_{S_{r}}(\bar{x}(t)) \leq 0$ for all $t \in\left[0, t_{0}+k^{*} \varepsilon\right]$; thus we have that $x(t) \in S_{r}$ for all $t \in\left[0, t^{*}\right]$.

The computation of a constant $C$ in the statement of the lemma, with the strengthened hypothesis $\left(\mathrm{F}^{*}\right)$ still in effect, follows in a routine way from the definition of the control $\bar{u}(\cdot)$, and is left to the reader.

It remains to show that $\left(\mathrm{F}^{*}\right)$ can in fact be assumed without loss of generality. To see this, note that for specified $T_{0}>0$, the global Lipschitz property of the extended dynamics implies that there exists $b>0$ such that all solutions $x(t)$ of $(1)$ with $x(0) \in S$ satisfy $\|x(t)\| \leq b$ for all $t \in\left[0, T_{0}\right]$. The above proof then goes through unchanged, upon setting

$$
H:=\max _{(x, u) \in\left\{b \bar{B}_{n}\right\} \times U}\|f(x, u)\|
$$

and replacing $t^{*}$ with $\min \left\{t^{*}, T_{0}\right\}$.

\subsection{Applications of the tracking lemma}

In this section, we shall employ the tracking lemma in order to derive regularity results on the family of problems $P_{r}(\tau, \alpha)$ as $r$ varies in $\left[0, r_{0}\right]$; here $P_{r}(\tau, \alpha)$ is the variant of $P(\tau, \alpha)$ wherein $S$ is replaced by the inner approximation $S_{r}$. It is a stepping stone to another type of approximation of $P(\tau, \alpha)$ in the next subsection, in which the state constraint will be removed. We denote the $S_{r}$-constrained value function by $V_{r}$; observe that in this notation, we could (but will not) write $V=V_{0}$. The first two results, which are of some independent interest, provide for local Lipschitz continuity of the $S_{r}$-constrained attainability map in a uniform way with respect to $r$, and immediate consequences involving continuity of each $V_{r}$. The third result (Prop. 3.13) deals with the nature of the convergence $V_{r} \rightarrow V$, and is important in our control feedback construction. 
Given $(\tau, \alpha) \in(-\infty, T] \times S_{r}$, we define $A_{S_{r}}(\tau, \alpha ; T)$, the $S_{r}$-constrained attainable set at time $T$, to be the set of points $x(T)=x(T ; \tau, \alpha, u(\cdot))$, where $u(\cdot)$ is any control such that $x(t ; \tau, \alpha, u(\cdot)) \in S_{r}$ for all $t \in[\tau, T]$. In all the results of this subsection and the next, conditions (S1) and (S2) will be assumed to hold.

Theorem 3.10. There exists $W>0$ such that for every $r \in\left[0, r_{0}\right]$,

$$
\left.\begin{array}{l}
\left(\tau_{0}, \alpha_{0}\right) \in[0, T] \times S_{r} \\
\left(\tau_{1}, \alpha_{1}\right) \in[0, T] \times S_{r}
\end{array}\right\} \Longrightarrow A_{S_{r}}\left(\tau_{0}, \alpha_{0} ; T\right) \subset A_{S_{r}}\left(\tau_{1}, \alpha_{1} ; T\right)+W\left[\left|\tau_{0}-\tau_{1}\right|+\left\|\alpha_{0}-\alpha_{1}\right\|\right] \bar{B}_{n} .
$$

Proof. Fix $r \in\left[0, r_{0}\right]$ and let $\left(\tau_{0}, \alpha_{0}\right) \in[0, T] \times S_{r}$, and let $u(\cdot)$ be a control such that

$$
x(t):=x\left(t ; \tau_{0}, \alpha_{0}, u(\cdot)\right) \in S_{r}
$$

on the interval $\left[\tau_{0}, T\right]$.

First consider an initial phase $\left(\tau_{1}, \alpha_{0}\right)$, where $0 \leq \tau_{1} \leq \tau_{0}$, and define the control

$$
\hat{u}(t):=\left\{\begin{array}{cl}
u\left(t+\tau_{0}-\tau_{1}\right) & \text { if } t \in\left[\tau_{1}, T-\tau_{0}+\tau_{1}\right] \\
\tilde{u}(t) & \text { if } t \in\left[T-\tau_{0}+\tau_{1}, T\right]
\end{array}\right.
$$

where $\tilde{u}(\cdot)$ is a control such that

$$
x\left(t ; T-\tau_{0}+\tau_{1}, x\left(T-\tau_{0}+\tau_{1}\right), \tilde{u}(\cdot)\right) \in S_{r} \quad \forall t \in\left[T-\tau_{0}+\tau_{1}, T\right] .
$$

Then the trajectory $\hat{x}(t):=x\left(t ; \tau_{1}, \alpha_{0}, \hat{u}(\cdot)\right)$ remains in $S_{r}$ on the interval $\left[\tau_{1}, T\right]$, and

$$
\|x(T)-\hat{x}(T)\| \leq Q\left\|\tau_{0}-\tau_{1}\right\|,
$$

where

$$
Q:=\max \{\|v\|: v \in f(S, U)\}
$$

Now consider the case $0 \leq \tau_{0} \leq \tau_{1} \leq T$. Then we simply define $\hat{u}(t):=u\left(t+\tau_{0}-\tau_{1}\right)$ on $\left[\tau_{1}, T\right]$. It follows that $\hat{x}(t) \in S_{r}$ on that interval, and again we obtain (63), which shows that the multifunction $A_{S_{r}}$ is Lipschitz with respect to the initial time $\tau$.

In order to verify local Lipschitzness of $A_{S_{r}}$ with respect to the initial state $\alpha$, fix $\tau_{0} \in[0, T]$, let $\alpha_{0} \in S_{r}$, and let $u(\cdot)$ be a control on $\left[\tau_{0}, T\right]$ such that the trajectory $x(t):=x\left(t ; \tau_{0}, \alpha_{0}, u(\cdot)\right)$ remains in $S_{r}$ on $\left[\tau_{0}, T\right]$.

In our interval-by-interval construction, which utilizes the tracking lemma, we will assume that $T-\tau_{0} \geq t^{*}$. The other case is easier and left to the reader.

Consider an initial phase $\left(\tau_{0}, \alpha_{1}\right)$ with $\alpha_{1} \in S_{r}$, and apply the same control $u(\cdot)$ on the interval $\left[\tau_{0}, \tau_{0}+t^{*}\right]$. By Gronwall's lemma,

$$
\left\|x\left(t ; \tau_{0}, \alpha_{0}, u(\cdot)\right)-x\left(t ; \tau_{0}, \alpha_{1}, u(\cdot)\right)\right\| \leq \tilde{K}\left\|\alpha_{0}-\alpha_{1}\right\| \quad \forall t \in\left[\tau_{0}, \tau_{0}+t^{*}\right],
$$

with $\tilde{K}:=\left(e^{K T}-1\right)$, where $K>0$ is a Lipschitz constant as in (F1) (with $\Gamma=S$ ). Therefore

$$
\max _{t \in\left[\tau_{0}, \tau_{0}+t^{*}\right]} d_{S_{r}}\left(x\left(t ; \tau_{0}, \alpha_{1}, u(\cdot)\right)\right) \leq \tilde{K}\left\|\alpha_{0}-\alpha_{1}\right\|,
$$

and the tracking lemma implies that there exists a control $\bar{u}(\cdot)$ on $\left[\tau_{0}, \tau_{0}+t^{*}\right]$ such that

$$
x\left(t ; \tau_{0}, \alpha_{1}, \bar{u}(\cdot)\right) \in S_{r} \quad \forall t \in\left[\tau_{0}, \tau_{0}+t^{*}\right]
$$


and

$$
\left\|x\left(t ; \tau_{0}, \alpha_{1}, u(\cdot)\right)-x\left(t ; \tau_{0}, \alpha_{1}, \bar{u}(\cdot)\right)\right\| \leq C \tilde{K}\left\|\alpha_{0}-\alpha_{1}\right\| \quad \forall t \in\left[\tau_{0}, \tau_{0}+t^{*}\right] .
$$

Then

$$
\left\|x\left(t ; \tau_{0}, \alpha_{0}, u(\cdot)\right)-x\left(t ; \tau_{0}, \alpha_{1}, \bar{u}(\cdot)\right)\right\| \leq(\tilde{K}+C \tilde{K})\left\|\alpha_{0}-\alpha_{1}\right\| \quad \forall t \in\left[\tau_{0}, \tau_{0}+t^{*}\right] .
$$

This procedure is now repeated, with $x\left(\tau_{0}+t^{*} ; \tau_{0}, \alpha_{0}, u(\cdot)\right)$ and $x\left(\tau_{0}+t^{*} ; \tau_{0}, \alpha_{1}, \bar{u}(\cdot)\right)$ taking over the roles played by $\alpha_{0}$ and $\alpha_{1}$, respectively. Accordingly, we extend the control $\bar{u}(\cdot)$ to the interval $\left[\tau_{0}+t^{*}, \tau_{0}+2 t^{*}\right]$, and obtain

$$
x\left(t ; \tau_{0}, \alpha_{1}, \bar{u}(\cdot)\right) \in S_{r} \quad \forall t \in\left[\tau_{0}+t^{*}, \tau_{0}+2 t^{*}\right]
$$

and

$$
\left\|x\left(t ; \tau_{0}, \alpha_{0}, u(\cdot)\right)-x\left(t ; \tau_{0}, \alpha_{1}, \bar{u}(\cdot)\right)\right\| \leq(\tilde{K}+C \tilde{K})^{2}\left\|\alpha_{0}-\alpha_{1}\right\| \quad \forall t \in\left[\tau_{0}+t^{*}, \tau_{0}+2 t^{*}\right] .
$$

Continuing in this way, we finally obtain

$$
x\left(t ; \tau_{0}, \alpha_{1}, \bar{u}(\cdot)\right) \in S_{r} \quad \forall t \in\left[\tau_{0}, T\right]
$$

and

$$
\left\|x\left(t ; \tau_{0}, \alpha_{0}, u(\cdot)\right)-x\left(t ; \tau_{0}, \alpha_{1}, \bar{u}(\cdot)\right)\right\| \leq(\tilde{K}+C \tilde{K})^{\tilde{m}}\|\alpha-\hat{\alpha}\| \quad \forall t \in\left[\tau_{0}, T\right],
$$

where $\bar{m}$ denotes the smallest positive integer $m$ such that $m t^{*} \geq T$. Upon combining (63) and (72), we obtain the result, with $W=Q+(\tilde{K}+C \tilde{K})^{\bar{m}}$.

The following consequence of the theorem is readily verified.

Corollary 3.11. There exists $\hat{K}>0$ such that for each $r \in\left[0, r_{0}\right], V_{r}$ is Lipschitz of rank $\hat{K}$ on $[0, T] \times S_{r}$.

Remark 3.12. In Section 1 we have exhibited an example where continuity of $V$ in a state constrained problem can fail; there both (S1) and (S2) were violated, and it was noted that state constrained tracking fails to hold. The following example is more subtle. The state constraint set is now

$$
S=\left\{\left(x_{1}, x_{2}\right):\left|x_{1}\right|+x_{2} \geq 0\right\} \cap \bar{B}_{2} .
$$

It is readily noted that $(\mathrm{S} 1)$ holds. Near the origin, the dynamics are given by

$$
\begin{aligned}
& \dot{x}_{1}=u, \quad U=[-2,2] \\
& \dot{x}_{2}=-1,
\end{aligned}
$$

and one can define the dynamics elsewhere in such a way that $(\mathrm{F} 1, \mathrm{~F} 2)$ hold and so that (S2) fails only at $(0,0)$. Then $S$ is weakly invariant, since (19) holds; the origin is not problematic in this regard, since $N_{S}^{P}(0,0)=$ $\{(0,0)\}$. We take the cost functional to be $\ell(x(1))=x_{1}(1)$.

For $i=1,2 \ldots$, denote $\alpha_{i}=\left(-\frac{1}{i},-\frac{1}{i}\right)$ and $\bar{\alpha}_{i}=\left(\frac{1}{i},-\frac{1}{i}\right)$. For each initial phase $\left(0, \alpha_{i}\right)$, the optimal control is $u(t) \equiv-2$, and so $V\left(0, \alpha_{i}\right)=-\frac{1}{i}-2$. On the other hand, for initial phases $\left(0, \bar{\alpha}_{i}\right)$, the optimal control is $u(t) \equiv 1$, and we obtain $V\left(0, \bar{\alpha}_{i}\right)=1+\frac{1}{i}$. Upon letting $i \rightarrow \infty$, we see that $V$ is discontinuous at $(0,0,0)$.

To see that the conclusion of the tracking lemma fails to hold in this example, consider an initial phase $\left(0, \bar{\alpha}_{i}\right)$ and apply the control $u(t) \equiv-2$. The resulting trajectory $\left(\frac{1}{i}-2 t,-t\right)$ immediately exits $S$, and then re-enters $S$ later. As $i \rightarrow \infty$, the amount of time the trajectory is exterior to $S$ decreases to zero, as does the maximal 
distance from $S$ of any point along the trajectory, during an initial time interval. Upon considering the nature of trajectories emanating from $\bar{\alpha}_{i}$ which remain in $S$ (they necessarily move "down and to the right"), one easily concludes that no constants $C$ and $t^{*}$ as in (56) exist.

It is not hard to see that for any $(\tau, \alpha) \in(-\infty, T] \times S_{r}$, one has

$$
V(\tau, \alpha) \leq V_{r}(\tau, \alpha)
$$

The reason for this is that the $S$-constrained problem $P(\tau, \alpha)$ admits more feasible trajectories than its $S_{r^{-}}$ constrained counterpart, $P_{r}(\tau, \alpha)$.

It is natural to ask whether the $S_{r}$-constrained value function, $V_{r}$, converges to the $S$-constrained value function $V$ in an appropriate uniform manner as $r$ becomes small. The following result confirms this.

Proposition 3.13. There exists $\hat{W}>0$ such that for each initial phase $(\tau, \alpha) \in[0, T] \times \operatorname{int}(S)$ and each $r \in\left[0, r_{0}\right]$ such that $r \leq d_{\hat{S}}(\alpha)$, one has

$$
V_{r}(\tau, \alpha) \leq V(\tau, \alpha)+\hat{W} r
$$

and so

$$
\left\|V_{r}(\tau, \alpha)-V(\tau, \alpha)\right\| \leq \hat{W} r .
$$

Proof. Consider $(\tau, \alpha)$ and $r$ as in the statement, and let $u(\cdot)$ be an optimal control in the $S$ constrained problem with initial phase $(\tau, \alpha)$. In view of the tracking lemma, there exist $t^{*}>0, C>0$ and a control $\bar{u}(\cdot)$ such that

$$
x(t ; 0, \alpha, \bar{u}(\cdot)) \in S_{r} \quad \forall t \in\left[\tau, \tau+t^{*}\right]
$$

and

$$
\|x(t ; 0, \alpha, u(\cdot))-x(t ; 0, \alpha, \bar{u}(\cdot))\| \leq C \max _{t \in\left[0, t^{*}\right]} d_{S_{r}}(x(t ; 0, \alpha, u(\cdot))) \quad \forall t \in\left[\tau, \tau+t^{*}\right]
$$

On the interval $\left[\tau+t^{*}, \tau+2 t^{*}\right]$, we consider the trajectory emanating from the point $x\left(\tau+t^{*} ; \tau, \alpha, \bar{u}(\cdot)\right)$, when the control $u(\cdot)$ is applied. Gronwall's inequality implies

$$
\left\|x\left(t ; \tau+t^{*}, x\left(\tau+t^{*} ; \tau, \alpha, \bar{u}(\cdot)\right), u(\cdot)\right)-x(t ; \tau, \alpha, u(\cdot))\right\| \leq C r \tilde{K} \quad \forall t \in\left[\tau+t^{*}, \tau+2 t^{*}\right],
$$

where $\tilde{K}>0$ is as in (64). We now reapply the tracking lemma and extend $\bar{u}(\cdot)$ to the interval $\left[\tau+t^{*}, \tau+2 t^{*}\right]$ in such a way that

$$
x(t ; 0, \alpha, \bar{u}(\cdot)) \in S_{r} \quad \forall t \in\left[\tau+t^{*}, \tau+2 t^{*}\right]
$$

and

$$
\left\|x(t ; \tau, \alpha, \bar{u}(\cdot))-x\left(t ; \tau+t^{*}, x\left(\tau+t^{*} ; \tau, \alpha, \bar{u}(\cdot)\right), u(\cdot)\right)\right\| \leq C^{2} r \tilde{K} \quad \forall t \in\left[\tau+t^{*}, \tau+2 t^{*}\right]
$$

Then

$$
\| x(t ; \tau, \alpha, \bar{u}(\cdot))-x\left(t ; \tau, \alpha, u(\cdot) \| \leq C r \tilde{K}+C^{2} r \tilde{K} \quad \forall t \in\left[\tau+t^{*}, \tau+2 t^{*}\right]\right.
$$


As in the proof of Theorem 3.10, let $\bar{m}$ be the smallest integer such that $m t^{*} \geq T$. By repeating the previous arguments, one then obtains

$$
\| x(t ; \tau, \alpha, \bar{u}(\cdot))-x\left(t ; \tau, \alpha, u(\cdot) \| \leq r \tilde{K} \sum_{i=1}^{\bar{m}} C^{i} \quad \forall t \in[\tau, T]\right.
$$

and $x(t ; \tau, \alpha, \bar{u}(\cdot)) \in S_{r}$ for all $t \in[\tau, T]$.

Denote a Lipschitz constant for $\ell(\cdot)$ on $S$ by $K_{\ell}$. Then

$$
\ell(x(T ; \tau, \alpha, \bar{u}(\cdot))) \leq \ell(x(T ; \tau, \alpha, u(\cdot)))+K_{\ell} r \tilde{K} \sum_{i=1}^{\bar{m}} C^{i},
$$

which implies

$$
V_{r}(\tau, \alpha) \leq V(\tau, \alpha)+r \hat{W}
$$

with $\hat{W}:=K_{\ell} \tilde{K} \sum_{i=1}^{\bar{m}} C^{i}$, completing the proof.

\subsection{Constraint removal}

A well known method of approximating the state constrained problem $P(\tau, \alpha)$ by a problem free of such constraints involves the imposition of a penalty term. A familiar instance of this involves analyzing a problem $P^{k}(\tau, \alpha)$ (where $k>0$ ) in which the goal is to minimize the cost

$$
\ell(x(T))+k \int_{\tau}^{T} \mathrm{~d}_{S}(x(t)) \mathrm{d} t
$$

over all trajectories satisfying only the requirement $x(\tau)=\alpha$. If we denote the value function in this problem by $V^{k}(\tau, \alpha)$, then for any $(\tau, \alpha) \in[0, T] \times S$ we clearly have $V^{k}(\tau, \alpha) \leq V(\tau, \alpha)$; hence, unlike $V_{r}$, the value function $V_{k}$ is a lower approximation of $V$. Under reasonable hypotheses on the data, one can show that $V^{k}(\tau, \alpha)$ converges to $V(\tau, \alpha)$ as $k \rightarrow \infty$; that is, as the penalty for being exterior to $S$ increases in severity. It is our purpose to develop a useful upper approximation of $V$ via constraint removal by a very different technique - one that involves modification of the dynamics near the boundary of $S$ and the exploitation of the properties we have already derived for problem $P_{r}(\tau, \alpha)$.

Fix $r \in\left(0, r_{0}\right)$, and suppose that $\alpha \in S$ is such that $0 \leq d_{\hat{S}}(\alpha)<r$, and consider the function $v(\cdot)$ occurring in Lemma 3.5. Since $v(\cdot)$ is a Lipschitz function defined on the compact set $Q\left(S ; r_{0}\right)$, there is a unique solution $x(\cdot)$ to the differential equation

$$
\dot{x}(t)=v(x(t)) \quad t \in\left[0, t^{0}(\alpha)\right),
$$

satisfying the initial condition $x(0)=\alpha$, where

$$
t^{0}(\alpha):=\sup \left\{t: d_{\hat{S}}(x(t)) \leq r_{0}\right\}
$$

In view of (41), the set $S_{r(\alpha)}$ is invariant (we can drop the use of "weakly" here), and so $x(t) \in S_{r(\alpha)}$ on the interval $\left[0, t^{0}(\alpha)\right)$.

We will require the following preparatory result. For convenience, we denote $\hat{\mu}:=\frac{\hat{\varepsilon} \mu}{3}$.

Lemma 3.14. One has

$$
t(\alpha):=\sup \left\{t: d_{\hat{S}}(x(t))=r\right\} \leq \frac{2 r}{\hat{\mu}} .
$$


Proof. Consider the absolutely continuous function $h:\left[0, t^{0}(\alpha)\right) \rightarrow \mathbb{R}$ given by $h(t):=d_{\hat{S}}(x(t))$. The lemma will follow immediately upon verification of the fact that

$$
\dot{h}(t) \geq \frac{\hat{\mu}}{2}, \quad t \in\left[0, t^{0}(\alpha)\right) \text { a.e. }
$$

On the interval $\left[0, t^{0}(\alpha)\right)$, consider the absolutely continuous function $g(t):=\Delta_{S_{r(\alpha)}}(x(t))$, and observe that on that interval one has $g(t)=-d_{\widehat{S_{r(\alpha)}}}(x(t))$. Consequently,

$$
h(t)=r(\alpha)-g(t)
$$

By a $C$-calculus chain rule (see Th. 2.3.2 in [17]), one has

$$
\dot{g}(t) \in\left\{\langle v(x(t)), \zeta\rangle: \zeta \in \partial_{C} \Delta_{S_{r(\alpha)}}(x(t))\right\}
$$

at every point in $\left[0, t^{0}(\alpha)\right)$ where $g(\cdot)$ is differentiable. In view of $(41)$ and $(53)$, one has

$$
\langle v(\alpha), \zeta\rangle \leq-\hat{\mu} \quad \forall \zeta \in \partial_{C} \Delta_{S_{r(\alpha)}}(\alpha) .
$$

Therefore, in view of the continuity of the function $t \rightarrow v(x(t))$ and the graph closedness of the multifunction $\partial_{C} \Delta_{S_{\hat{r}}}(\cdot)$, there exists $\tilde{t}=\tilde{t}(\alpha) \in\left(0, t^{0}(\alpha)\right)$ such that $\dot{g}(t) \leq-\frac{\hat{\mu}}{2}$ almost everywhere on the interval $[0, \tilde{t}]$. Since this argument can be extended with $x(\tilde{t})$ taking over the role of $\alpha$, we conclude that $\dot{g}(t) \leq-\frac{\hat{\mu}}{2}$ almost everywhere on $\left[0, t^{0}(\alpha)\right)$. Then $(87)$ yields $(86)$.

We now introduce a modification of the original dynamics. Let $K_{v}$ denote the Lipschitz rank of $v(\cdot)$ on $Q\left(S ; r_{0}\right)$. For each component $v_{i}(\cdot)(i=1,2, \ldots, n)$, let

$$
\tilde{v}_{i}(x)=\min _{y \in \operatorname{bdry}(S)}\left\{v_{i}(y)+K_{v}\|y-x\|\right\} .
$$

Then $\tilde{v}(\cdot): \mathbb{R}^{n} \rightarrow \mathbb{R}^{n}$ is a function which is globally Lipschitz of rank $K_{v}$, and such that $\tilde{v}(\cdot)=v(\cdot)$ on bdry $(S)$. For a given value $r \in\left[0, r_{0}\right)$, we define a multifunction $F_{r}$ on $\mathbb{R}^{n}$ as follows.

$$
F_{r}(x):=\left\{\begin{array}{cl}
f(x, U) & \text { if } x \in S_{r} \\
\frac{d_{\hat{S}}(x) f(x, U)+d_{S_{r}}(x) v(x)}{d_{\hat{S}}(x)+d_{S_{r}}(x)} & \text { if } x \in S \backslash S_{r} \\
\tilde{v}(x) & \text { if } x \in \hat{S} .
\end{array}\right.
$$

Hence, $F_{r}(\cdot)$ agrees with $f(\cdot, U)$ on the inner approximation $S_{r}$, and is a certain convex combination of $f(\cdot, U)$ and $v(\cdot)$ on $S \backslash S_{r}$. On $\hat{S}$, we have $F_{r}(\cdot)=\{\tilde{v}(\cdot)\}$.

Lemma 3.15. The following properties hold for each $r \in\left(0, r_{0}\right)$ :

(a) $F_{r}(x)$ is compact and convex for every $x \in \mathbb{R}^{n}$.

(b) One has

$$
v(x) \in F_{r}(x) \subset f(x, U) \quad \forall x \in S \backslash S_{r}
$$

(c) For every $r \in\left[0, r_{0}\right]$, the multifunction $F_{r}(\cdot)$ is globally Lipschitz of rank $\hat{K}:=\max \left\{K, K_{v}\right\}$.

(d) For every $(\tau, \alpha) \in \mathbb{R} \times \mathbb{R}^{n}$, there exists a trajectory (i.e. an absolutely continuous arc) $x(\cdot)$ satisfying the differential inclusion 


$$
\dot{x}(t) \in F_{r}(x(t)) \quad \text { a.e. }
$$

on $[\tau, \infty)$, such that $x(\tau)=\alpha$.

(e) For any $(\tau, \alpha) \in(-\infty, T] \times \mathbb{R}^{n}$, the set of trajectories $x(\cdot)$ of (91) on the interval $[\tau, T]$, satisfying $x(\tau)=\alpha$, is sequentially compact in the uniform topology.

(f) The set $S$ is strongly invariant with respect to $F_{r}$; that is, for every $(\tau, \alpha) \in \mathbb{R} \times S$, every trajectory $x(\cdot)$ of (91) with $x(\tau)=\alpha$ satisfies $x(t) \in S$ for all $t \geq \tau$.

We remark that the values of $F_{r}$ outside $S$ are not explicitly relevant in our constructions, but it is convenient to have $F_{r}$ globally well-behaved as asserted above. Parts $(\mathrm{a}-\mathrm{c})$ are quite immediate, while $(\mathrm{d}-\mathrm{e})$ are standard facts about differential inclusions; note that the required growth condition holds due to the global Lipschitz nature of $F_{r}$. Part (f) holds because $F_{r}$ is Lipschitz and satisfies the tangency condition

$$
F_{r}(x) \subset T_{S}^{C}(x) \quad \forall x \in S,
$$

by virtue of (40). The proof that this suffices for strong invariance is to be found in either [13] or [17].

Now consider the optimal control problem $\tilde{P}_{r}(\tau, \alpha)$, with dynamics provided by the differential inclusion (91), where the goal is to minimize the cost functional $\ell(x(T))$, and where no state constraint is present. Note well however, that the strong invariance asserted in part (f) of the lemma shows that for initial phases $(\tau, \alpha) \in$ $[0, T] \times S$, the state constraint $x(t) \in S$ on $[\tau, T]$ is satisfied implicitly. In view of parts (d) and (e) of the preceding lemma, for every such initial phase $(\tau, \alpha)$, an optimal trajectory exists. We go on to denote the value function in this problem by $\tilde{V}_{r}:(-\infty, T] \times \mathbb{R}^{n} \rightarrow \mathbb{R}$. Note that part (b) of the above lemma implies

$$
V(\tau, \alpha) \leq \tilde{V}_{r}(\tau, \alpha) \quad \forall(\tau, \alpha) \in(-\infty, T] \times S .
$$

The next proposition provides for the approximation of the value function $V$ of our original $S$-constrained problem, by $\tilde{V}_{r}$.

Proposition 3.16. Assume that $r_{0}>0$ in the previous results is taken small enough so that $\frac{2 r_{0}}{\hat{\mu}}<T$. Then there exists $E>0$ such that for every $r \in\left(0, r_{0}\right)$ and every $(\tau, \alpha) \in[0, T] \times S$, one has

$$
\tilde{V}_{r}(\tau, \alpha) \leq V(\tau, \alpha)+E r,
$$

and so

$$
\left\|V(\tau, \alpha)-\tilde{V}_{r}(\tau, \alpha)\right\| \leq E r .
$$

Proof. We will break the proof into three cases.

Case 1: $(\tau, \alpha) \in\left[T-\frac{2 r}{\hat{\mu}}, T\right] \times S$.

Let $x_{1}(\cdot)$ and $x_{2}(\cdot)$ be optimal trajectories in problems $\tilde{P}_{r}(\tau, \alpha)$ and $P(\tau, \alpha)$, respectively. Then

$$
\begin{aligned}
0 & \leq \tilde{V}_{r}(\tau, \alpha)-V(\tau, \alpha) \\
& =\ell\left(x_{1}(T)\right)-\ell\left(x_{2}(T)\right) \\
& =\ell\left(x_{1}(T)\right)-\ell(\alpha)+\ell(\alpha)-\ell\left(x_{2}(T)\right) \\
& \leq K_{\ell}\left\|x_{1}(T)-\alpha\right\|+K_{\ell}\left\|x_{2}(T)-\alpha\right\| \\
& \leq 2 K_{\ell} Q(T-\tau) \leq \frac{4 K_{\ell} Q r}{\hat{\mu}} .
\end{aligned}
$$


Note that the norm bound $Q$ on $f(S, U)$ also serves as a norm bound on $F_{r}(S)$, due to (90). Hence the last inequality above is valid, since both $x_{1}(\cdot)$ and $x_{2}(\cdot)$ remain in $S$. This is true of the first trajectory by strong invariance, and of the second by definition of $P(\tau, \alpha)$.

Case 2: $(\tau, \alpha) \in\left[0, T-\frac{2 r}{\hat{\mu}}\right] \times S_{r}$.

In this case we have

$$
\tilde{V}_{r}(\tau, \alpha) \leq V_{r}(\tau, \alpha) \leq V(\tau, \alpha)+\hat{W} r,
$$

where the second inequality holds due to Proposition 3.13. We conclude that

$$
0 \leq \tilde{V}_{r}-V(\tau, \alpha) \leq \hat{W} r
$$

Case 3: $(\tau, \alpha) \in\left[0, T-\frac{2 r}{\hat{\mu}}\right] \times\left\{S \backslash S_{r}\right\}$.

In view of Lemma 3.14, there exists a trajectory $x(\cdot)$ of the differential inclusion $(91)$ on the interval $[\tau, t(\alpha)]$, emanating from the initial phase $(\tau, \alpha)$, such that $x(t(\alpha)) \in S_{r}$, where

$$
t(\alpha)<\tau+\frac{2 r}{\hat{\mu}} \leq T
$$

Note that

$$
\|x(t(\alpha))-\alpha\| \leq Q(t(\alpha)-\tau) \leq \frac{2 Q r}{\hat{\mu}}
$$

Suppose that the extended (for $t$ beyond $t(\alpha)$ ) trajectory $x(\cdot)$ is optimal in problem $P_{r}(t(\alpha), x(t(\alpha))$ ). Then one has

$$
\begin{aligned}
\tilde{V}_{r}(\tau, \alpha) & \leq V_{r}(t(\alpha), x(t(\alpha))) \\
& \leq V(t(\alpha), x(t(\alpha)))+\hat{W} r \\
& \leq V(\tau, \alpha)+\hat{K}\|(t(\alpha)-\tau, x(t(\alpha))-\alpha)\|+\hat{W} r \\
& \leq V(\tau, \alpha)+\hat{K}\left(\frac{2 r}{\hat{\mu}}+\frac{2 Q r}{\hat{\mu}}\right)+\hat{W} r \\
& =V(\tau, \alpha)+r \hat{K}\left(\frac{2+2 Q}{\hat{\mu}}+\hat{W}\right)
\end{aligned}
$$

Hence, we have

$$
0 \leq \tilde{V}_{r}(\tau, \alpha)-V(\tau, \alpha) \leq r \hat{K}\left(\frac{2+2 Q}{\hat{\mu}}+\hat{W}\right)
$$

Upon setting

$$
E=\max \left\{\frac{4 K_{\ell} Q}{\hat{\mu}}, \hat{K}\left(\frac{2+2 Q}{\hat{\mu}}+\hat{W}\right)\right\}
$$

the proof is complete.

We will require one more lemma prior to providing our main result in the next section. 
Lemma 3.17. Let $r \in\left(0, r_{0}\right)$. Then there exists $T(r)>0$ such that if $\alpha \in S$ and $u_{\alpha} \in U$ are such that $f\left(\alpha, u_{\alpha}\right) \in F_{r}(\alpha)$, then the (unique) solution $x_{\alpha}(\cdot)$ of the differential equation

$$
\dot{x}(t)=f\left(x(t), u_{\alpha}\right), \quad t \geq 0,
$$

with $x(0)=\alpha$, satisfies $x_{\alpha}(t) \in S$ for all $t \in[0, T(r)]$.

Proof. Suppose the claim was false. Then there would exist sequences $\alpha_{i} \in S, u_{\alpha_{i}} \in U$, such that

$$
t_{i}:=\max \left\{t \geq 0: x_{\alpha_{i}}(t) \in S \forall t \in[0, t]\right\} \rightarrow 0 .
$$

By compactness, we can assume that $\alpha_{i} \rightarrow \hat{\alpha} \in S$ and $u_{\alpha_{i}} \rightarrow \hat{u} \in U$. It is clear that neccessarily $\hat{\alpha} \in \operatorname{bdry}(S)$, and therefore $F_{r}(\hat{\alpha})=\{v(\hat{\alpha})\}$. Now, Lemma 3.6 implies that for all sufficiently large $i$ one has

$$
\alpha_{i}+W(v(\hat{\alpha}) ; \tilde{\varepsilon}) \subset S .
$$

Fix such an $i$, and consider the curves

$$
x_{\alpha_{i}}(t)=\alpha_{i}+\int_{0}^{t} f\left(x_{\alpha_{i}}(s), u_{\alpha_{i}}\right) \mathrm{d} s
$$

and

$$
z_{i}(t)=\alpha_{i}+\int_{0}^{t} f\left(\alpha_{i}, u_{\alpha_{i}}\right) \mathrm{d} s=\alpha_{i}+t f\left(\alpha_{i}, u_{\alpha_{i}}\right) .
$$

Observe that if $t \leq 1$ (say), then

$$
\left\|x_{\alpha_{i}}(t)-z_{i}(t)\right\| \leq t K \kappa
$$

where

$$
\kappa:=\max _{\alpha \in S} \max _{t \in[0,1]}\left\|x_{\alpha}(t)-\alpha\right\|>0 .
$$

By increasing $i$ if necessary, $f\left(\alpha_{i}, u_{\alpha_{i}}\right)$ approximates $v(\hat{\alpha})$ sufficiently closely that we have, by $(99)$,

$$
z_{i}(t) \in \alpha_{i}+W\left(v(\hat{\alpha}) ; \frac{\tilde{\varepsilon}}{4}\right) \subset S \quad \forall t \in\left[0, \frac{\tilde{\varepsilon}}{4}\right] .
$$

We deduce that

$$
x_{\alpha_{i}}(t) \in \alpha_{i}+W\left(v\left(\alpha_{i}\right) ; \frac{\tilde{\varepsilon}}{2}\right) \subset S \quad \forall t \in[0, \hat{T}],
$$

where

$$
\hat{T}:=\min \left\{\frac{\tilde{\varepsilon}}{4}, \frac{\tilde{\varepsilon}}{4 K \kappa}\right\} .
$$

Since these estimates are applicable for every large $i$, we have contradicted $t_{i} \rightarrow 0$, which completes the proof. 


\section{NeAR-OPtimal Universal CONTROL FEEDBACK IN STATE CONSTRAined OPTIMAL CONTROL}

\subsection{Main result}

The main result of this section is the following.

Theorem 4.1. Let $\varepsilon>0$ be given. Then there exists a control feedback $k(t, x)$ along with a positive number $\delta$, such that for every initial phase $(\tau, \alpha) \in[0, T) \times S$ and any partition $\pi$ of $[\tau, T]$ with $\operatorname{diam}(\pi)<\delta$, the associated $\pi$-trajectory $x_{\pi}$ satisfying $x_{\pi}(\tau)=\alpha$ also satisfies

$$
x_{\pi}(t) \in S \quad \forall t \in[\tau, T]
$$

and

$$
\ell\left(x_{\pi}(T)\right) \leq V(\tau, \alpha)+\varepsilon .
$$

Here $\operatorname{diam}(\pi):=\max \left\{t_{i+1}-t_{i}: i=0,1, \ldots, N_{\pi}-1\right\}$. The theorem asserts that if the partition is of sufficiently small diameter (or the sampling rate is sufficiently high), the control feedback $k(t, x)$ produces a $\pi$-trajectory which is $\varepsilon$-optimal universally in the $S$-constrained problem $P(\tau, \alpha)$ universally for initial phases in the generalized rectangle $[0, T] \times S$.

\section{Remark 4.2.}

(a) To the best of our knowledge, in optimal control problems without state constraints, the construction of universal near-optimal discretization procedures, or of discontinuous near-optimal control feedback laws, goes back to Krasovskiı̌ [24] and was elaborated upon in [25] and [26]. See also Krasovskiı̌ and Subbotin [27,28], as well as Subbotin [40]; the later reference contains further bibliographic references. Other relevant references include Berkovitz [5], Subbotina [42], Cannarsa and Frankowska [6], Rowland and Vinter [36], as well as Ancona and Bressan [1]. The latter reference (which is concerned with stabilizability) is of interest because discretization of the dynamics is not required; instead, the type of discontinuous feedbacks considered have the property that the corresponding dynamics admit classical solutions. A control feedback construction technique for pursuit problems is found in Clarke et al. $[14,15]$; see also Garnysheva and Subbotin [21] as well as Subbotin [40].

(b) The control feedback construction technique in Nobakhtian and Stern [30] (again, without state constraints), as well as the stabilization results of Clarke et al. [10], involve "proximal aiming" to a family of "shells" constructed from a value function or Lyapunov function. (For background on the proximal aiming method, in somewhat simpler contexts, we refer the reader to [13] and [17], while the reference Sontag [38] provides general background on the stabilizability problem.) The shell-based approach was simplified (in the stabilizability setting) by Rifford in [31,32], and [33] by utilizing semiconcavity properties of the quadratic infimal convolution of a Lyapunov function. We shall adapt this method (which was in fact implicit and used in [11] and [14]) in proving Theorem 4.1.

(c) The universality property of the control feedback produced in Theorem 4.1 is an important distinction from control feedbacks which are effective only from a given initial phase, and in a sense, the weakening of "optimal" to " $\varepsilon$-optimal for any given $\varepsilon>0$ " in Theorem 4.1 below can be viewed as the price paid for universality in our constructive method, albeit a small one in any practical sense. Whether this price is truly unavoidable is an open question ${ }^{4}$. On the other hand, Subbotina [41] (see also Krasovskiur and Subbotin [28]) have provided an example of a fixed duration differential game which does not possess a universal saddle point, under hypotheses which imply the existence of a saddle point for each individual initial phase.

\footnotetext{
${ }^{4}$ It has come to our attention that Subbotina [43] has recently investigated this issue.
} 
(d) Theorem 4.1 is related to recent work of Ishii and Koike [23], who adapted the methodology of Clarke et al. [11] (which dealt with stabilization) in order to construct an $\varepsilon$-optimal universal control feedback in an infinite horizon discounted cost optimal control problem with a state contraint. Specifically, in [23] it is proven that for given $\varepsilon>0$, there is a mesh diameter (or sampling rate) and a corresponding control feedback, defined for that particular mesh diameter, which achieves $\varepsilon$-optimality in a universal manner. This is in sharp contrast to the control feedback provided in Theorem 4.1, which is universally operative for all small mesh diameters.

Our main task will be to prove the following.

Proposition 4.3. Let $\varepsilon>0$ be given, and fix $r \in\left(0, r_{0}\right)$, where $\frac{2 r_{0}}{\hat{\mu}}<T$, as in Proposition 3.16. Then there exists a control feedback $k(t, x)=k_{r}(t, x)$ along with a positive number $\delta$, such that for every initial phase $(\tau, \alpha) \in[0, T) \times S$ and any partition $\pi$ of $[\tau, T]$ with $\operatorname{diam}(\pi)<\delta$, the associated $\pi$-trajectory $x_{\pi}$ satisfying $x_{\pi}(\tau)=\alpha$ also satisfies

$$
x_{\pi}(t) \in S \quad \forall t \in[\tau, T]
$$

and

$$
\ell\left(x_{\pi}(T)\right) \leq \tilde{V}_{r}(\tau, \alpha)+\frac{\varepsilon}{2}
$$

Let us show how the main result follows from this proposition.

Proof of Theorem 4.1. In Proposition 3.16, take $r$ so small that $E r<\frac{\varepsilon}{2}$. Then we see that in Proposition 4.3, the control feedback $k(t, x)$ produces, for partitions of $[\tau, T]$ with sufficiently small diameter, a $\pi$-trajectory emanating from the initial phase $(\tau, \alpha) \in[0, T] \times S$ such that (102) holds as well as

$$
\ell\left(x_{\pi}(T)\right) \leq V(\tau, \alpha)+\varepsilon
$$

and $k(t, x)$ has this property for any such $(\tau, \alpha)$. The theorem now follows.

Let us extend the domain of $\tilde{V}_{r}:(-\infty, T] \times \mathbb{R}^{n} \rightarrow \mathbb{R}$ to all of $\mathbb{R} \times \mathbb{R}^{n}$ by setting $\tilde{V}_{r}(t, \cdot)=\ell(\cdot)$ for $t \geq T$. The following lemma is a consequence of a standard fact about value functions when no state constraint is present; see $[17]$.

Lemma 4.4. For each $r \in\left(0, r_{0}\right)$, the extended value function $\tilde{V}_{r}$ is locally Lipschitz on $\mathbb{R} \times \mathbb{R}^{n}$.

Proof of Proposition 4.3. We shall first verify the following.

Claim. Without loss of generality, we can assume that $\ell$ is globally bounded below.

In order to understand the legitimacy of this assumption in the statement of the proposition, first note that there exists $D>0$ such that for any initial phase $(\tau, \alpha) \in[0, T] \times S$ and any trajectory $x(\cdot)$ of $(91)$ with $x(\tau)=\alpha$, one has $\|x(t)\| \leq D$ for all $t \in[\tau, T]$. Now consider the function

$$
\tilde{\ell}(x):=\max \{\ell(x), M\}
$$

where

$$
M:=\min _{x \in D \bar{B}_{n}} \ell(x)
$$

Then $\tilde{\ell}$ is locally Lipschitz on $\mathbb{R}^{n}$ and agrees with $\ell$ on $D \bar{B}_{n}$. It is clear that replacing $\ell$ with $\tilde{\ell}$ has no effect on the content of the proposition, which verifies the claim. 
- Simplified notation: For simplicity of exposition, we will write $\tilde{V}_{r}=\tilde{V}$ from this point on.

The existence of optimal trajectories implies that for every $\left(t_{0}, x_{0}\right) \in[-\infty, T] \times \mathbb{R}^{n}$, there exists a trajectory $x(\cdot)$ on $\left[t_{0}, T\right)$ such that $x\left(t_{0}\right)=x_{0}$ and

$$
\tilde{V}(t, x(t)) \leq \tilde{V}\left(t_{0}, x_{0}\right) \quad \forall t \in\left[t_{0}, T\right] .
$$

(In fact, this holds in equality form, but it is the inequality which is of use to us.) This property, termed weak decrease of $\tilde{V}$ on $(-\infty, T) \times \mathbb{R}^{n}$, is equivalent to the proximal Hamilton-Jacobi inequality

$$
\bar{h}_{r}(x, \theta, \zeta) \leq 0 \quad \forall(\theta, \zeta) \in \partial_{P} \tilde{V}(t, x), \quad \forall(t, x) \in(-\infty, T) \times \mathbb{R}^{n} ;
$$

see $[13,17]$. Here $\bar{h}_{r}: \mathbb{R}^{n} \times \mathbb{R} \times \mathbb{R}^{n} \rightarrow \mathbb{R}$ denotes the extended lower Hamiltonian for $F_{r}$, given by

$$
\bar{h}_{r}(x, \theta, \zeta):=\theta+h_{r}(x, \zeta)
$$

where at present, instead of (13), we write

$$
h_{r}(x, p):=\min _{v \in F_{r}(x)}\langle v, p\rangle
$$

For a given parameter value $\lambda>0$, the quadratic inf-convolution of $\tilde{V}$ is given by

$$
\tilde{V}_{\lambda}(t, x)=\min _{\left(t^{\prime}, x^{\prime}\right) \in \mathbb{R} \times \mathbb{R}^{n}}\left\{\tilde{V}\left(t^{\prime}, x^{\prime}\right)+\left\|(t, x)-\left(t^{\prime}, x^{\prime}\right)\right\|^{2}\right\} .
$$

Recalling the discussion in Section 2.3 and bearing in mind that $\tilde{V}$ is locally Lipschitz (due to Lem. 4.4) and globally bounded below (by the above claim), we have that $\tilde{V}_{\lambda}$ is semiconcave on $\mathbb{R} \times \mathbb{R}^{n}$, and that the minimum in (106) is attained.

Let us temporarily fix real numbers $a^{\prime}, a^{\prime \prime}, T^{\prime \prime}, T^{\prime}, M^{\prime}, M^{\prime \prime}$ such that

$$
a^{\prime}<a^{\prime \prime}<0<T^{\prime \prime}<T^{\prime}<T
$$

and

$$
0<M^{\prime}<M^{\prime \prime}
$$

The following approximation lemma will be required:

Lemma 4.5. Let $\varepsilon_{1}>0$ be given. Then for all sufficiently large $\lambda>0$, one has

$$
\begin{gathered}
\left|\tilde{V}_{\lambda}(t, x)-\tilde{V}(t, x)\right| \leq \varepsilon_{1} \quad \forall(t, x) \in\left[a^{\prime \prime}, T^{\prime}\right] \times M^{\prime} \bar{B}_{n}, \\
\bar{h}_{r}(x, \theta, \zeta) \leq \varepsilon_{1} \quad \forall(\theta, \zeta) \in \partial_{P} \tilde{V}_{\lambda}(t, x), \quad \forall(t, x) \in\left[a^{\prime \prime}, T^{\prime}\right] \times M^{\prime} \bar{B}_{n},
\end{gathered}
$$

and

$$
\bar{h}_{r}(x, \theta, \zeta) \leq \varepsilon_{1} \quad \forall(\theta, \zeta) \in \partial_{L} \tilde{V}_{\lambda}(t, x), \quad \forall(t, x) \in\left[0, T^{\prime \prime}\right] \times M^{\prime} B_{n} .
$$


Proof. Let

$$
Z=\max _{(t, x) \in\left[a^{\prime}, T\right] \times M^{\prime} \bar{B}_{n}} \tilde{V}(t, x) .
$$

The fact that $\tilde{V}_{\lambda}$ is majorized by $\tilde{V}$ implies the following: For every $(t, x) \in\left[a^{\prime \prime}, T^{\prime}\right] \times M^{\prime} \bar{B}_{n}$ and every $\lambda>0$, there exists $(\bar{t}, \bar{x}) \in \mathbb{R} \times \mathbb{R}^{n}$ such that

$$
\tilde{V}_{\lambda}(t, x)=\tilde{V}(\bar{t}, \bar{x})+\lambda\|(t, x)-(\bar{t}, \bar{x})\|^{2} \leq \tilde{V}(t, x) \leq Z \text {. }
$$

In view of the claim we have verified, we can assume that $\tilde{V}$ is globally bounded below; we denote such a lower bound by $c$. Then

$$
\|(t, x)-(\bar{t}, \bar{x})\|^{2} \leq \frac{Z-c}{\lambda}
$$

It follows that $\lambda$ may be taken large enough to ensure that for every $(t, x) \in\left[a^{\prime \prime}, T^{\prime}\right] \times M^{\prime} \bar{B}_{n}$, one has

$$
(\bar{t}, \bar{x}) \in\left[a^{\prime}, T\right] \times M^{\prime \prime} \bar{B}_{n} .
$$

Therefore

$$
\tilde{V}(\bar{t}, \bar{x}) \geq \tilde{V}(t, x)-L_{\tilde{V}}\|(t, x)-(\bar{t}, \bar{x})\|,
$$

where $L_{\tilde{V}}$ is a Lipschitz constant for $\tilde{V}$ on $\left[a^{\prime}, T\right] \times M^{\prime \prime} \bar{B}_{n}$; here we again used Lemma 4.4. Since $\tilde{V}_{\lambda}(t, x) \geq$ $\tilde{V}(\bar{t}, \bar{x})$, it follows that

$$
\tilde{V}_{\lambda}(t, x) \geq \tilde{V}(t, x)-L_{\tilde{V}}\|(t, x)-(\bar{t}, \bar{x})\| .
$$

Then, since $\tilde{V}_{\lambda}(t, x) \leq \tilde{V}(t, x)$, we arrive at

$$
\left|\tilde{V}_{\lambda}(t, x)-\tilde{V}(t, x)\right| \leq L_{\tilde{V}} \sqrt{\frac{Z-c}{\lambda}} \quad \forall(t, x) \in\left[0, T^{\prime}\right] \times M^{\prime} \bar{B}_{n}
$$

and (107) follows.

In order to prove (108), let $(t, x) \in\left[a^{\prime \prime}, T^{\prime}\right] \times M^{\prime} \bar{B}_{n}$ and $(\theta, \zeta) \in \partial_{P} \tilde{V}_{\lambda}(t, x)$. Then $(\theta, \zeta) \in \partial_{P} \tilde{V}(\bar{t}, \bar{x})$, where $(\bar{t}, \bar{x})$ is as above, with $\lambda$ taken large enough that (112) holds. We also note that by (105), we have

$$
\bar{h}_{r}(\bar{x}, \theta, \zeta)=\theta+\langle\bar{v}, \zeta\rangle \leq 0,
$$

for a certain $\bar{v} \in F_{r}(\bar{x})$. Now let $\hat{v}$ be the (unique) closest point in $F_{r}(x)$ to $\bar{v}$. Then

$$
\begin{aligned}
\bar{h}_{r}(x, \theta, \zeta) & \leq \theta+\langle\hat{v}, \zeta\rangle \\
& =\theta+\langle\bar{v}, \zeta\rangle+\langle\hat{v}-\bar{v}, \zeta\rangle \\
& =\bar{h}_{r}(\bar{x}, \theta, \zeta)+\langle\hat{v}-\bar{v}, \zeta\rangle \\
& \leq\langle\hat{v}-\bar{v}, \zeta\rangle \\
& \leq \hat{K}\|\zeta\|\|\bar{x}-x\| \\
& \leq \hat{K}\|\zeta\| \sqrt{\frac{Z-c}{\lambda}} \quad \text { by (111). }
\end{aligned}
$$


Since $\tilde{V}$ is Lipschitz of $\operatorname{rank} L_{\tilde{V}}$ on $\left[a^{\prime}, T\right] \times M^{\prime \prime} \bar{B}_{n}$, one has

$$
\|\zeta\| \leq\|(\theta, \zeta)\| \leq L_{\tilde{V}}
$$

and for sufficiently large $\lambda$, (108) follows.

Finally, equation (109) follows immediately from (108), by the definition of the $L$-subdifferential.

We return now to the proof of the proposition. For a given parameter value $\lambda$, a control feedback $k: \mathbb{R} \times \mathbb{R}^{n} \rightarrow$ $U$ is defined as follows.

- Let $(t, x) \in \mathbb{R} \times \mathbb{R}^{n}$.

- If $x \in S$, arbitrarily choose $(\theta, \zeta) \in \partial_{L} \tilde{V}_{\lambda}(t, x)$, and then set $k(t, x)=u \in U$ such that $f(x, u) \in F_{r}(x)$ and

$$
\bar{h}_{r}(x, \theta, \zeta)=\theta+\langle f(x, u), \zeta\rangle
$$

- If $x \notin S$, take $k(t, x)$ to be any element of $U$.

Remark 4.6. It is the $L$-subdifferential of $\tilde{V}$ that figures in the control feedback's definition, and not the $P$-subdifferential. The advantage of this choice is that $\partial_{L} \tilde{V}(t, x) \neq \phi$ at all points $(t, x)$, whereas the possible emptiness of $\partial_{P} \tilde{V}(t, x)$ would be problematic in our ensuing construction. It is also worth noting that in the construction of a $\pi$-trajectory associated with the control feedback $k(t, x)$, the choice of $(\theta, \zeta) \in \partial_{L} \tilde{V}_{\lambda}\left(t_{i}, x_{i}\right)$ does not need to be "remembered" at the next node $\left(t_{i+1}, x_{i+1}\right)$, so in the case of an "on-line" procedure, it suffices to calculate an arbitrary $L$-subgradient when a given phase is reached.

Let $\varepsilon_{1}>0$ be given, let $M^{\prime}>\max _{x \in S}\|x\|$, specify $M^{\prime \prime}, a^{\prime}, a^{\prime \prime}, T^{\prime \prime}$ and $T^{\prime}$ as above, and take $\lambda>0$ large enough that $(108,109)$ hold.

For a given partition $\pi$ of $[\tau, T]$ as in (10), we shall consider the $\pi$-trajectory $x_{\pi}(\cdot)$ on $[\tau, T]$ generated by the control feedback defined above, with $x_{\pi}(\tau)=\alpha$. Observe that

- Lemma 3.17 guarantees $x_{\pi}(t) \in S$ on $[0, T]$, if $\operatorname{diam}(\pi)<T(r)$.

Since $\tilde{V}_{\lambda}$ is semiconcave on $\mathbb{R} \times \mathbb{R}^{n}$, one has

$$
-\tilde{V}_{\lambda}\left(t^{\prime}, x^{\prime}\right)+\tilde{V}_{\lambda}(t, x)+\lambda\left\|\left(t^{\prime}, x^{\prime}\right)-(t, x)\right\|^{2} \geq\left\langle(\theta, \zeta),\left(t^{\prime}, x^{\prime}\right)-(t, x)\right\rangle \quad \forall(\theta, \zeta) \in \partial_{P}\left(-\tilde{V}_{\lambda}\right)(t, x)
$$

for every $(t, x)$ and $\left(t^{\prime}, x^{\prime}\right)$ in $\mathbb{R} \times \mathbb{R}^{n}$. Then in view of the semiconcavity property (9) combined with the $C$-calculus fact (3), we have

$$
-\tilde{V}_{\lambda}\left(t^{\prime}, x^{\prime}\right)+\tilde{V}_{\lambda}(t, x)+\lambda\left\|\left(t^{\prime}, x^{\prime}\right)-(t, x)\right\| \geq\left\langle-(\theta, \zeta),\left(t^{\prime}, x^{\prime}\right)-(t, x)\right\rangle \quad \forall(\theta, \zeta) \in \partial_{L} \tilde{V}_{\lambda}(t, x)
$$

for every $(t, x)$ and $\left(t^{\prime}, x^{\prime}\right)$.

Let

$$
i^{*}:=\max \left\{i: t_{i} \leq T^{\prime \prime}\right\}
$$

We denote $\left(t_{0}, x_{0}\right)=(\tau, \alpha)$. Pick $\left(\theta_{0}, \zeta_{0}\right) \in \partial_{L} \tilde{V}_{\lambda}\left(t_{0}, x_{0}\right)$. Recall that $Q$ denotes a norm bound on the set $f(S, U)$, $K$ is the Lipschitz constant occurring in (F1), and $L_{\tilde{V}}$ is a Lipschitz constant for $\tilde{V}$ on the set $\left[a^{\prime}, T\right] \times M^{\prime \prime} \bar{B}_{n}$. 
Then, bearing (109) and (115) in mind, for $t$ in the interval $\left[\tau, t_{1}\right]=\left[t_{0}, t_{1}\right]$ we have

$$
\begin{aligned}
\tilde{V}_{\lambda}\left(t, x_{\pi}(t)\right)-\tilde{V}_{\lambda}\left(t_{0}, x_{0}\right) & \leq\left\langle\left(\theta_{0}, \zeta_{0}\right),\left(t, x_{\pi}(t)\right)-\left(t_{0}, x_{0}\right)\right\rangle+\lambda\left\|\left(t, x_{\pi}(t)\right)-\left(t_{0}, x_{0}\right)\right\|^{2} \\
& =\left\langle\left(\theta_{0}, \zeta_{0}\right), \int_{t_{0}}^{t}\left(1, f\left(x_{\pi}(s), k\left(t_{0}, x_{0}\right)\right)\right) \mathrm{d} s\right\rangle+\lambda\left\|\left(t, x_{\pi}(t)\right)-\left(t_{0}, x_{0}\right)\right\|^{2} \\
& \leq\left\langle\left(\theta_{0}, \zeta_{0}\right), \int_{t_{0}}^{t}\left(1, f\left(x_{0}, k\left(t_{0}, x_{0}\right)\right)\right) \mathrm{d} s\right\rangle+L_{\tilde{V}} K Q\left(t-t_{0}\right)^{2}+\lambda\left\|\left(t, x_{\pi}(t)\right)-\left(t_{0}, x_{0}\right)\right\|^{2} \\
& \leq \varepsilon_{1}\left(t-t_{0}\right)+L_{\tilde{V}} K Q\left(t-t_{0}\right)^{2}+\lambda\left\|\left(t, x_{\pi}(t)\right)-\left(t_{0}, x_{0}\right)\right\|^{2} .
\end{aligned}
$$

It follows that

$$
\tilde{V}_{\lambda}\left(t_{1}, x_{\pi}\left(t_{1}\right)\right)-\tilde{V}_{\lambda}\left(t_{0}, x_{0}\right) \leq \varepsilon_{1}\left(t_{1}-t_{0}\right)+\left[L_{\tilde{V}} K Q+\lambda(1+Q)^{2}\right]\left(t_{1}-t_{0}\right)^{2} .
$$

We now repeat this calculation on the next interval, $\left[t_{1}, t_{2}\right]$. Upon picking $\left.\left(\theta_{1}, \zeta_{1}\right) \in \partial_{L} \tilde{V}_{\lambda}\left(t_{1}, x_{\pi}\left(t_{1}\right)\right)\right)$, one obtains

$$
\tilde{V}_{\lambda}\left(t_{2}, x_{\pi}\left(t_{2}\right)\right)-\tilde{V}_{\lambda}\left(t_{1}, x_{\pi}\left(t_{1}\right)\right) \leq \varepsilon_{1}\left(t_{2}-t_{1}\right)+\left[L_{\tilde{V}} K Q+\lambda(1+Q)^{2}\right]\left(t_{2}-t_{1}\right)^{2} .
$$

Upon combining this with the inequality obtained on the prior interval, we get

$$
\tilde{V}_{\lambda}\left(t_{2}, x_{\pi}\left(t_{2}\right)\right)-\tilde{V}_{\lambda}\left(t_{0}, x_{0}\right) \leq \varepsilon_{1}\left(t_{2}-t_{0}\right)+\left[L_{\tilde{V}} K Q+\lambda(1+Q)^{2}\right]\left(\left(t_{1}-t_{0}\right)^{2}+\left(t_{2}-t_{1}\right)^{2}\right) .
$$

Continuing this process, we eventually arrive at

$$
\tilde{V}_{\lambda}\left(t_{i^{*}}, x_{\pi}\left(t_{i^{*}}\right)\right)-\tilde{V}_{\lambda}\left(t_{0}, x_{0}\right) \leq \varepsilon_{1}\left(t_{i^{*}}-t_{0}\right)+\left[L_{\tilde{V}} K Q+\lambda(1+Q)^{2}\right] \operatorname{diam}(\pi)\left(t_{i^{*}}-t_{0}\right) .
$$

Then

$$
\tilde{V}_{\lambda}\left(t_{i^{*}}, x_{\pi}\left(t_{i^{*}}\right)\right)-\tilde{V}_{\lambda}\left(t_{0}, x_{0}\right) \leq \varepsilon_{1} T+\left[L_{\tilde{V}} K Q+\lambda(1+Q)^{2}\right] T \operatorname{diam}(\pi),
$$

and therefore

$$
\tilde{V}_{\lambda}\left(T, x_{\pi}(T)\right)-\tilde{V}_{\lambda}\left(t_{0}, x_{0}\right) \leq \varepsilon_{1} T+\left[L_{\tilde{V}} K Q+\lambda(1+Q)^{2}\right] T \operatorname{diam}(\pi)+K_{\ell} Q\left[T-T^{\prime \prime}+\operatorname{diam}(\pi)\right] .
$$

Summarizing, the parameter $\lambda>0$ is taken large enough so that (107)-(109) hold for a pre-specified $\varepsilon_{1}>0$. Then we obtain

$$
\tilde{V}\left(T, x_{\pi}(T)\right)-\tilde{V}\left(t_{0}, x_{0}\right) \leq \frac{\varepsilon}{2}
$$

by adjusting $T^{\prime \prime}$ to be near $T$, and by taking $\varepsilon_{1}$ and $\operatorname{diam}(\pi)$ sufficiently small (in addition to insisting that $\operatorname{diam}(\pi)<T(r))$. This concludes the proof.

Remark 4.7. Theorem 4.1 holds true as stated if the local Lipschitz assumption on the cost functional $\ell$ is relaxed to mere continuity. To understand this claim, suppose $\ell$ were only continuous on $\mathbb{R}^{n}$, let $\theta>0$, and consider the mollifier of $\ell$ given by

$$
\ell_{\theta}(x)=\int_{\mathbb{R}^{n}} \ell(x+\theta y) \omega(y) \mathrm{d} y,
$$

where $\omega: \mathbb{R}^{n} \rightarrow[0, \infty)$ is a function in $C^{\infty}\left(\mathbb{R}^{n}\right)$ with support in $\bar{B}_{n}$ such that

$$
\int_{\mathbb{R}^{n}} \omega(y) \mathrm{d} y=1 .
$$


Then standard arguments concerning regularization of continuous functions yield that $\ell_{\theta} \in C^{\infty}\left(\mathbb{R}^{n}\right)$. (There is no inherent benefit to replacing $\ell$ with a smooth function; Lipschitzness of the replacement suffices for our purposes.) Furthermore, for any given $\beta>0, \theta$ may be chosen small enough to ensure that

$$
\left|\ell_{\theta}(x)-\ell(x)\right| \leq \beta \quad \forall x \in S .
$$

It readily follows that $V$ is uniformly approximated by $V_{\theta}$ on $[0, T] \times S$ to arbitrary tolerance, where $V_{\theta}$ is the value function obtained by replacing $\ell$ with $\ell_{\theta}$. Since Theorem 4.1 involves only near (as opposed to exact) optimality, this clearly verifies the claim made.

\subsection{Robustness}

In this section we will prove that there exists a control feedback $\tilde{k}(t, x)$ which is robust with respect to state measurement errors which are small in an appropriate sense, when the partition in the discretization scheme has sufficiently small diameter (as in Th. 4.1), and in addition is required to be "reasonably uniform" in a sense to be made clear; see also Remark 4.9 below. The perturbed system under study is modeled by

$$
\dot{x}(t)=f(x(t), \tilde{k}(t, x(t)+p(t)))
$$

where the function $p(\cdot)$ represents the observational error present in applying the control feedback.

Let an initial phase $(\tau, \alpha) \in(-\infty, T] \times \mathbb{R}^{n}$ be specified. Then given a partition

$$
\pi=\left\{\tau=t_{0}, t_{1}, \ldots, t_{N_{\pi}}=T\right\}
$$

of $[\tau, T]$, the $\pi$-trajectory $x_{\pi}$ on $[\tau, T]$ obtained in the model $(120)$ is the curve satisfying the following intervalby-interval dynamics: Upon setting $x_{0}=\alpha$, on the interval $\left[t_{0}, t_{1}\right], x_{\pi}$ is the classical solution of

$$
\dot{x}_{\pi}(t)=f\left(x_{\pi}(t), k\left(t_{0}, x_{0}+p_{0}\right)\right), \quad x_{\pi}\left(t_{0}\right)=x_{0}, \quad t \in\left(t_{0}, t_{1}\right) .
$$

We then set $x_{1}:=x_{\pi}\left(t_{1}\right)$, and restart the process on the next interval:

$$
\dot{x}_{\pi}(t)=f\left(x_{\pi}(t), k\left(t_{1}, x_{1}+p_{1}\right)\right), \quad x_{\pi}\left(t_{1}\right)=x_{1}, \quad t \in\left(t_{1}, t_{2}\right) .
$$

We continue in this way through the last interval $\left[t_{N_{\pi}-1}, t_{N_{\pi}}\right]$. Here the continuous function $x_{\pi}(t)$ is the actual state of the system at time $t$, and the values $x_{i}+p_{i}$ correspond to the inexact measurements used to generate the piecewise constant control function in the scheme.

We have the following robust version of Theorem 4.1. The result allows for erroneous measurements of the state giving values exterior to $S$, while the $\pi$-trajectory that is generated is near-optimal and remains in $S$.

Theorem 4.8. Let $\varepsilon>0$ be given. Then there exists a control feedback $\tilde{k}(t, x)$ along with a positive number $\delta_{0}$ such that the following holds: for every $\delta \in\left(0, \delta_{0}\right)$ there exists $E(\delta)>0$ with the property that for any partition $\pi$ of $[0, T]$ having

$$
\frac{\delta}{2} \leq t_{i+1}-t_{i} \leq \delta \quad \forall i=0,1, \ldots, N_{\pi}-1
$$

the error bounds

$$
\left\|p_{i}\right\|<E(\delta) \quad \forall i=0,1, \ldots, N_{\pi}-1
$$

imply that for any initial phase $(\tau, \alpha) \in[0, T) \times S$, the $\pi$-trajectory $x_{\pi}$ with $x_{\pi}(\tau)=\alpha$, satisfies

$$
x_{\pi}(t) \in S \quad \forall t \in[\tau, T]
$$


and

$$
\ell\left(x_{\pi}(T)\right) \leq V(\tau, \alpha)+\varepsilon
$$

Proof. For each $x \in \mathbb{R}^{n}$, choose $s(x) \in \operatorname{proj}_{S}(x)$. We will show that the control feedback

$$
\tilde{k}(t, x):=k(t, s(x))
$$

has the required properties, where $k(t, x)$ is the control feedback occurring in Theorem 4.1 , by modifying the estimates in the proof of Proposition 4.3. As before, one has that Lemma 3.17 implies $x_{\pi}(t) \in S$ on $[0, T]$, if $\operatorname{diam}(\pi)<T(r)$.

In addition to the notations employed in the proof of Theorem 4.1, let us denote by $L_{\tilde{V}_{\lambda}}$ a Lipschitz constant for $\tilde{V}_{\lambda}$ on an open neighborhood of $[0, T] \times S$, for a value of the parameter $\lambda$ which is subsequently to be specified.

- We will assume at the outset that the error terms satisfy $\left\|p_{i}\right\| \leq 1$ for each $i$.

Our estimates below will make use of the obvious fact that for any points $x \in S$ and $p \in \mathbb{R}^{n}$, one has

$$
\|s(x+p)-x\| \leq 2\|p\|
$$

Consider the first interval $\left[\tau, t_{1}\right]=\left[t_{0}, t_{1}\right]$, and choose $\left(\theta_{0}, \zeta_{0}\right) \in \partial_{L} \tilde{V}_{\lambda}\left(t_{0}, s\left(x_{0}+p_{0}\right)\right)$. For $t \in\left[t_{0}, t_{1}\right]$, one has

$$
\begin{aligned}
\tilde{V}_{\lambda}\left(t, x_{\pi}(t)\right)-\tilde{V}_{\lambda}\left(t_{0}, x_{0}\right) \leq & \tilde{V}_{\lambda}\left(t, x_{\pi}(t)\right)-\tilde{V}_{\lambda}\left(t_{0}, s\left(x_{0}+p_{0}\right)\right)+2 L_{\tilde{V}_{\lambda}}\left\|p_{0}\right\| \\
\leq & \left\langle\left(\theta_{0}, \zeta_{0}\right),\left(t, x_{\pi}(t)\right)-\left(t_{0}, s\left(x_{0}+p_{0}\right)\right)\right\rangle+\lambda\left\|\left(t, x_{\pi}(t)\right)-\left(t_{0}, s\left(x_{0}+p_{0}\right)\right)\right\|^{2} \\
& +2 L_{\tilde{V}_{\lambda}}\left\|p_{0}\right\| .
\end{aligned}
$$

It is readily noted that

$$
\left\|\left(t, x_{\pi}(t)\right)-\left(t_{0}, s\left(x_{0}+p_{0}\right)\right)\right\| \leq\left\|\left(t, x_{\pi}(t)\right)-\left(t_{0}, x_{0}\right)\right\|+2\left\|p_{0}\right\|
$$

and

$$
\left\|(t, x(t))-\left(t_{0}, x_{0}\right)\right\| \leq(1+Q)\left(t-t_{0}\right) .
$$

Then

$$
\left\|\left(t, x_{\pi}(t)\right)-\left(t_{0}, s\left(x_{0}+p_{0}\right)\right)\right\|^{2} \leq(1+Q)^{2}\left(t-t_{0}\right)^{2}+4\left\|p_{0}\right\|^{2}+4(1+Q)\left(t-t_{0}\right)\left\|p_{0}\right\|,
$$

and since $\left\|p_{0}\right\| \leq 1$, it follows that

$$
\left\|\left(t, x_{\pi}(t)\right)-\left(t_{0}, s\left(x_{0}+p_{0}\right)\right)\right\|^{2} \leq(1+Q)^{2}\left(t-t_{0}\right)^{2}+4\left\|p_{0}\right\|+4(1+Q)\left(t-t_{0}\right)\left\|p_{0}\right\| .
$$

We also have

$$
\left\langle\left(\theta_{0}, \zeta_{0}\right),\left(t, x_{\pi}(t)\right)-\left(t_{0}, s\left(x_{0}+p_{0}\right)\right)\right\rangle \leq\left\langle\left(\theta_{0}, \zeta_{0}\right),\left(t, x_{\pi}(t)\right)-\left(t_{0}, x_{0}\right)\right\rangle+2 L_{\tilde{V}_{\lambda}}\left\|p_{0}\right\| .
$$

Combining the above facts, we obtain

$$
\begin{aligned}
\tilde{V}_{\lambda}\left(t, x_{\pi}(t)\right)-\tilde{V}_{\lambda}\left(t_{0}, x_{0}\right) \leq & \left\langle\left(\theta_{0}, \zeta_{0}\right),\left(t, x_{\pi}(t)\right)-\left(t_{0}, x_{0}\right)\right\rangle+\lambda(1+Q)^{2}\left(t-t_{0}\right)^{2} \\
& +4\left(L_{\tilde{V}_{\lambda}}+\lambda\right)\left\|p_{0}\right\|+4 \lambda(1+Q)\left(t-t_{0}\right)\left\|p_{0}\right\| .
\end{aligned}
$$


One also has

$$
\begin{aligned}
\left\langle\left(\theta_{0}, \zeta_{0}\right),\left(t, x_{\pi}(t)\right)-\left(t_{0}, x_{0}\right)\right\rangle= & \left\langle\left(\theta_{0}, \zeta_{0}\right), \int_{t_{0}}^{t}\left(1, f\left(x_{\pi}(s), k\left(t_{0}, s\left(x_{0}+p_{0}\right)\right)\right) \mathrm{d} s\right\rangle\right. \\
\leq & \left\langle\left(\theta_{0}, \zeta_{0}\right), \int_{t_{0}}^{t}\left(1, f\left(x_{0}, k\left(t_{0}, s\left(x_{0}+p_{0}\right)\right)\right)\right) \mathrm{d} s\right\rangle+L_{\tilde{V}} K Q\left(t-t_{0}\right)^{2} \\
\leq & \left\langle\left(\theta_{0}, \zeta_{0}\right), \int_{t_{0}}^{t}\left(1, f\left(s\left(x_{0}+p_{0}\right), k\left(t_{0}, s\left(x_{0}+p_{0}\right)\right)\right) \mathrm{d} s\right\rangle+L_{\tilde{V}} K Q\left(t-t_{0}\right)^{2}\right. \\
& +2\left(t-t_{0}\right) L_{\tilde{V}} K\left\|p_{0}\right\| \\
\leq & \varepsilon_{1}\left(t-t_{0}\right)+L_{\tilde{V}} K Q\left(t-t_{0}\right)^{2}+2\left(t-t_{0}\right) L_{\tilde{V}} K\left\|p_{0}\right\| .
\end{aligned}
$$

We therefore arrive at

$$
\begin{aligned}
\tilde{V}_{\lambda}\left(t_{1}, x_{\pi}\left(t_{1}\right)\right)-\tilde{V}_{\lambda}\left(t_{0}, x_{0}\right) \leq & \varepsilon_{1}\left(t_{1}-t_{0}\right)+\left[2 L_{\tilde{V}} K+4 \lambda(1+Q)\right]\left(t_{1}-t_{0}\right)\left\|p_{0}\right\| \\
& +4\left(L_{\tilde{V}_{\lambda}}+\lambda\right)\left\|p_{0}\right\|+\left[L_{\tilde{V}} K Q+\lambda(1+Q)^{2}\right]\left(t_{1}-t_{0}\right)^{2} .
\end{aligned}
$$

Similarly, on the next interval, $\left[t_{1}, t_{2}\right]$, after picking $\left(\theta_{1}, \zeta_{1}\right) \in \partial_{L} \tilde{V}_{\lambda}\left(t_{1}, s\left(x_{\pi}\left(t_{1}\right)\right)+p_{1}\right)$, we obtain

$$
\begin{aligned}
\tilde{V}_{\lambda}\left(t_{2}, x_{\pi}\left(t_{2}\right)\right)-\tilde{V}_{\lambda}\left(t_{1}, x_{\pi}\left(t_{1}\right)\right) \leq & \varepsilon_{1}\left(t_{2}-t_{1}\right)+\left[2 L_{\tilde{V}} K+4 \lambda(1+Q)\right]\left(t_{2}-t_{1}\right)\left\|p_{1}\right\| \\
& +4\left(L_{\tilde{V}_{\lambda}}+\lambda\right)\left\|p_{0}\right\|+\left[L_{\tilde{V}} K Q+\lambda(1+Q)^{2}\right]\left(t_{1}-t_{0}\right)^{2} .
\end{aligned}
$$

Upon combining this with the inequality obtained on $\left[t_{0}, t_{1}\right]$, one obtains

$$
\begin{aligned}
\tilde{V}_{\lambda}\left(t_{2}, x_{\pi}\left(t_{2}\right)\right)-\tilde{V}_{\lambda}\left(t_{0}, x_{0}\right) \leq & \varepsilon_{1}\left(t_{2}-t_{0}\right)+\left[2 L_{\tilde{V}} K+4 \lambda(1+Q)\right]\left[\left(t_{1}-t_{0}\right)\left\|p_{0}\right\|+\left(t_{2}-t_{1}\right)\left\|p_{1}\right\|\right] \\
& +4\left(L_{\tilde{V}_{\lambda}}+\lambda\right)\left[\left\|p_{0}\right\|+\left\|p_{1}\right\|\right] \\
& +\left[L_{\tilde{V}} K Q+\lambda(1+Q)^{2}\right]\left(\left(t_{1}-t_{0}\right)^{2}+\left(t_{2}-t_{1}\right)^{2}\right) .
\end{aligned}
$$

Continuing in this way, we eventually obtain

$$
\begin{aligned}
\tilde{V}_{\lambda}\left(t_{i^{*}}, x_{\pi}\left(t_{i^{*}}\right)\right)-\tilde{V}_{\lambda}\left(t_{0}, x_{0}\right) \leq & \varepsilon_{1}\left(t_{i^{*}}-t_{0}\right)+\left[2 T L_{\tilde{V}} K+4 T \lambda(1+Q)\right] \max _{0 \leq i \leq N_{\pi}-1}\left\|p_{i}\right\| \\
& +4\left(L_{\tilde{V}_{\lambda}}+\lambda\right) \sum_{0 \leq i \leq N_{\pi}-1}\left\|p_{i}\right\|+\left[L_{\tilde{V}} K Q+\lambda(1+Q)^{2}\right] T \operatorname{diam}(\pi) .
\end{aligned}
$$

It follows that

$$
\begin{aligned}
\tilde{V}_{\lambda}\left(t_{i^{*}}, x_{\pi}\left(t_{i^{*}}\right)\right)-\tilde{V}_{\lambda}\left(t_{0}, x_{0}\right) \leq & \varepsilon_{1} T+\left[2 T L_{\tilde{V}} K+4 T \lambda(1+Q)\right] \max _{0 \leq i \leq N_{\pi}-1}\left\|p_{i}\right\| \\
& +4\left(L_{\tilde{V}_{\lambda}}+\lambda\right) \sum_{0 \leq i \leq N_{\pi}-1}\left\|p_{i}\right\|+\left[L_{\tilde{V}} K Q+\lambda(1+Q)^{2}\right] T \operatorname{diam}(\pi) .
\end{aligned}
$$

Then, similarly to the argument following (118),

$$
\begin{aligned}
\tilde{V}_{\lambda}\left(T, x_{\pi}(T)\right)-\tilde{V}_{\lambda}\left(t_{0}, x_{0}\right) \leq & \varepsilon_{1} T+\left[2 T L_{\tilde{V}} K+4 T \lambda(1+Q)\right] \max _{0 \leq i \leq N_{\pi}-1}\left\|p_{i}\right\| \\
& +4\left(L_{\tilde{V}_{\lambda}}+\lambda\right) \sum_{0 \leq i \leq N_{\pi}-1}\left\|p_{i}\right\| \\
& +\left[L_{\tilde{V}} K Q+\lambda(1+Q)^{2}\right] T \operatorname{diam}(\pi)+K_{\ell} Q\left[T-T^{\prime \prime}+\operatorname{diam}(\pi)\right] .
\end{aligned}
$$


We can arrange that

$$
\varepsilon_{1} T+\left[L_{\tilde{V}} K Q+\lambda(1+Q)^{2}\right] T \operatorname{diam}(\pi)+K_{\ell} Q\left[T-T^{\prime \prime}+\operatorname{diam}(\pi)\right] \leq \frac{\varepsilon}{8},
$$

and (by (107)) that

$$
\tilde{V}_{\lambda}(t, x)-\tilde{V}(t, x) \leq \frac{\varepsilon}{8} \quad \forall[0, T] \times S,
$$

by suitably adjusting $\lambda, \varepsilon_{1}, T^{\prime \prime}$, and by insisting that $\operatorname{diam}(\pi)<\delta_{0}$ for a sufficiently small $\delta_{0}>0$; we also take $\delta_{0}<T(r)$ to ensure that $x_{\pi}$ remains in $S$. Note that the choice of the parameter $\lambda$ determines a Lipschitz constant $L_{\tilde{V}_{\lambda}}$.

Let us now consider the other terms above, which include the measurement errors $p_{i}$, and involve the constant $L_{\tilde{V}_{\lambda}}$.

One has

$$
\max _{0 \leq i \leq N_{\pi}-1}\left\|p_{i}\right\|<\frac{\varepsilon}{16 L_{\tilde{V}} K+32 T \lambda(1+Q)} \Longrightarrow\left[2 T L_{\tilde{V}} K+4 T \lambda(1+Q)\right] \max _{0 \leq i \leq N_{\pi}-1}\left\|p_{i}\right\| \leq \frac{\varepsilon}{8} .
$$

Now recall that we are dealing with partitions $\pi$ which satisfy the condition(123). It follows that

$$
N_{\pi} \delta<2 T \text {. }
$$

We also have

$$
\sum_{0 \leq i \leq N_{\pi}-1}\left\|p_{i}\right\| \leq N_{\pi} \max _{0 \leq i \leq N_{\pi}-1}\left\|p_{i}\right\|
$$

These two observations readily yield the implication

$$
\max _{0 \leq i \leq N_{\pi}-1}\left\|p_{i}\right\|<\frac{\varepsilon \delta}{64 T\left(L_{\tilde{V}_{\lambda}}+\lambda\right)} \Longrightarrow 4\left(L_{\tilde{V}_{\lambda}}+\lambda\right) \sum_{0 \leq i \leq N_{\pi}-1}\left\|p_{i}\right\| \leq \frac{\varepsilon}{8} .
$$

We therefore set

$$
E(\delta):=\min \left\{1, \frac{\varepsilon}{16 T L_{\tilde{V}} K+32 T \lambda(1+Q)}, \frac{\varepsilon \delta}{64 T\left(L_{\tilde{V}_{\lambda}}+\lambda\right)}\right\},
$$

and require that $\left\|p_{i}\right\| \leq E(\delta)$ for each $i$; that is, equation (124). Note in particular that this bound incorporates the condition that the error terms satisfy $\left\|p_{i}\right\| \leq 1$, which we have been assuming throughout. Then (127) and (128) imply

$$
\left[2 T L_{\tilde{V}} K+4 T \lambda(1+Q)\right] \max _{0 \leq i \leq N_{\pi}-1}\left\|p_{i}\right\|+4\left(L_{\tilde{V}_{\lambda}}+\lambda\right) \sum_{0 \leq i \leq N_{\pi}-1}\left\|p_{i}\right\| \leq \frac{\varepsilon}{4}
$$

Summarizing, with $\lambda, \varepsilon_{1}, T^{\prime \prime}$ and $\delta_{0}$ adjusted as above, and with (124) holding, where the partitions satisfy (123) and with $E(\delta)$ defined as above, we obtain

$$
\tilde{V}\left(T, x_{\pi}(T)\right)-\tilde{V}\left(t_{0}, x_{0}\right) \leq \frac{\varepsilon}{2} .
$$

We have therefore arrived at a conclusion analagous to that reached in Proposition 4.3; namely, equation (119), as required. 
Remark 4.9. The fact that partitions with sufficiently small diameter are required in both Theorems 4.1 and 4.8 is quite natural, since this is what is needed in order for the decrease property (as manifested by proximal Hamilton-Jacobi inequalities) to come to bear in a discretized scheme such as ours. On the other hand, as was pointed out in [10] and Sontag [39] (with both references dealing with robust feedback stabilization via a shell-based approach), the near-uniformity of partitions posited in (123) serves the purpose of precluding a possible "chattering phenomenon" which could otherwise occur in the presence of state measurement errors.

Note also that the lower bound $\frac{\delta}{2}$ in (123) was used for simplicity; the result can be reformulated if $\frac{\delta}{2}$ is replaced by $g(\delta)$, where $g$ is any function such that $0<g(\delta)<\delta$ for all small $\delta>0$.

\section{REFERENCES}

[1] F. Ancona and A. Bressan, Patchy vector fields and asymptotic stabilization. ESAIM: COCV 4 (1999) 445-471.

[2] N.N. Barabanova and A.I. Subbotin, On continuous evasion strategies in game theoretic problems on the encounter of motions. Prikl. Mat. Mekh. 34 (1970) 796-803.

[3] N.N. Barabanova and A.I. Subbotin, On classes of strategies in differential games of evasion. Prikl. Mat. Mekh. 35 (1971) 385-392.

[4] M. Bardi and I. Capuzzo-Dolcetta, Optimal Control and Viscosity Solutions of Hamilton-Jacobi-Bellman Equations. Birkhäuser, Boston (1997).

[5] L.D. Berkovitz, Optimal feedback controls. SIAM J. Control Optim. 27 (1989) 991-1006.

[6] P. Cannarsa and H. Frankowska, Some characterizations of optimal trajectories in control theory. SIAM J. Control Optim. 29 (1991) 1322-1347.

[7] I. Capuzzo-Dolcetta and P.L. Lions, Hamilton-Jacobi equations with state constraints. Trans. Amer. Math. Soc. 318 (1990) 643-683.

[8] F.H. Clarke, Optimization and Nonsmooth Analysis. Wiley-Interscience, New York (1983). Republished as Vol. 5 of Classics in Appl. Math. SIAM, Philadelphia (1990).

[9] F.H. Clarke, Methods of Dynamic and Nonsmooth Optimization, Vol. 57 of CBMS-NSF Regional Conference Series in Applied Mathematics. SIAM, Philadelphia (1989).

[10] F.H. Clarke, Yu.S. Ledyaev, L. Rifford and R.J. Stern, Feedback stabilization and Lyapunov functions. SIAM J. Control Optim. 39 (2000) 25-48.

[11] F.H. Clarke, Yu.S. Ledyaev, E.D. Sontag and A.I. Subbotin, Asymptotic controllability implies control feedback stabilization. IEEE Trans. Automat. Control 42 (1997) 1394.

[12] F.H. Clarke, Yu.S. Ledyaev and R.J. Stern, Proximal analysis and control feedback construction. Proc. Steklov Inst. Math. 226 (2000) 1-20.

[13] F.H. Clarke, Yu.S. Ledyaev, R.J. Stern and P.R. Wolenski, Qualitative properties of trajectories of control systems: A survey. J. Dynam. Control Systems 1 (1995) 1-48.

[14] F.H. Clarke, Yu.S. Ledyaev and A.I. Subbotin, Universal feedback strategies for differential games of pursuit. SIAM J. Control Optim. 35 (1997) 552-561.

[15] F.H. Clarke, Yu.S. Ledyaev and A.I. Subbotin, Universal positional control. Proc. Steklov Inst. Math. 224 (1999) $165-186$. Preliminary version: Preprint CRM-2386. Univ. de Montréal (1994).

[16] F.H. Clarke, Yu.S. Ledyaev and R.J. Stern, Complements, approximations, smoothings and invariance properties. J. Convex Anal. 4 (1997) 189-219.

[17] F.H. Clarke, Yu.S. Ledyaev, R.J. Stern and P.R. Wolenski, Nonsmooth Analysis and Control Theory. Springer-Verlag, New York, Grad. Texts in Math. 178 (1998).

[18] F.H. Clarke, R.J. Stern and P.R. Wolenski, Proximal smoothness and the lower- $C^{2}$ property. J. Convex Anal. 2 (1995) $117-145$.

[19] F. Forcellini and F. Rampazzo, On nonconvex differential inclusions whose state is constrained in the closure of an open set. Applications to dynamic programming. Differential and Integral Equations 12 (1999) 471-497.

[20] H. Frankowska and F. Rampazzo, Filippov's and Filippov-Wazewski's theorems on closed domains. J. Differential Equations 161 (2000) 449-478.

[21] G.G. Garnysheva and A.I. Subbotin, Suboptimal universal strategies in a game-theoretic time-optimality problem. Prikl. Mat. Mekh. 59 (1995) 707-713.

[22] J.-B. Hiriart-Urruty, New concepts in nondifferentiable programming. Bull. Soc. Math. France 60 (1979) 57-85.

[23] H. Ishii and S. Koike, On ع-optimal controls for state constraint problems. Ann. Inst. H. Poincaré Anal. Linéaire 17 (2000) 473-502.

[24] N.N. Krasovskiǔ, Differential games. Approximate and formal models. Mat. Sb. (N.S.) 107 (1978) 541-571.

[25] N.N. Krasovskiı̌, Extremal aiming and extremal displacement in a game-theoretical control. Problems Control Inform. Theory 13 (1984) 287-302.

[26] N.N. Krasovskiū, Control of dynamical systems. Nauka, Moscow (1985). 
[27] N.N. Krasovskiı̌ and A.I. Subbotin, Positional Differential Games. Nauka, Moscow (1974). French translation: Jeux Différentielles. Mir, Moscou (1979).

[28] N.N. Krasovskiǔ and A.I. Subbotin, Game-Theoretical Control Problems. Springer-Verlag, New York (1988).

[29] P. Loewen, Optimal Control via Nonsmooth Analysis. CRM Proc. Lecture Notes Amer. Math. Soc. 2 (1993).

[30] S. Nobakhtian and R.J. Stern, Universal near-optimal control feedbacks. J. Optim. Theory Appl. 107 (2000) 89-123.

[31] L. Rifford, Problèmes de Stabilisation en Théorie du Contrôle, Doctoral Thesis. Univ. Claude Bernard Lyon 1 (2000).

[32] L. Rifford, Stabilisation des systèmes globalement asymptotiquement commandables. C. R. Acad. Sci. Paris 330 (2000) 211-216.

[33] L. Rifford, Existence of Lipschitz and semiconcave control-Lyapunov functions. SIAM J. Control Optim. (to appear).

[34] R.T. Rockafellar, Clarke's tangent cones and boundaries of closed sets in $\mathbb{R}^{n}$. Nonlinear Anal. 3 (1979) 145-154.

[35] R.T. Rockafellar, Favorable classes of Lipschitz continuous functions in subgradient optimization, in Nondifferentiable Optimization, edited by E. Nurminski. Permagon Press, New York (1982).

[36] J.D.L. Rowland and R.B. Vinter, Construction of optimal control feedback controls. Systems Control Lett. 16 (1991) $357-357$.

[37] M. Soner, Optimal control problems with state-space constraints I. SIAM J. Control Optim. 24 (1986) 551-561.

[38] E.D. Sontag, Mathematical Control Theory, 2nd Ed.. Springer-Verlag, New York, Texts in Appl. Math. 6 (1998).

[39] E.D. Sontag, Clock and insensitivity to small measurement errors. ESAIM: COCV 4 (1999) 537-557.

[40] A.I. Subbotin, Generalized Solutions of First Order PDE's. Birkhäuser, Boston (1995).

[41] N.N. Subbotina, Universal optimal strategies in positional differential games. Differential Equations 19 (1983) $1377-1382$.

[42] N.N. Subbotina, The maximum principle and the superdifferential of the value function. Problems Control Inform. Theory $\mathbf{1 8}$ (1989) 151-160.

[43] N.N. Subbotina, On structure of optimal feedbacks to control problems, Preprints of the eleventh IFAC International Workshop, Control Applications of Optimization, edited by V. Zakharov (2000).

[44] R.B. Vinter, Optimal Control. Birkhäuser, Boston (2000). 